\title{
ON SEPARATION BY LOCALLY TESTABLE AND LOCALLY THRESHOLD TESTABLE LANGUAGES
}

\author{
THOMAS PLACE, LORIJN VAN ROOIJEN, AND MARC ZEITOUN
}

LaBRI, Bordeaux University, France

e-mail address: firstname.lastname@labri.fr

\begin{abstract}
A separator for two languages is a third language containing the first one and disjoint from the second one. We investigate the following decision problem: given two regular input languages, decide whether there exists a locally testable (resp. a locally threshold testable) separator. In both cases, we design a decision procedure based on the occurrence of special patterns in automata accepting the input languages. We prove that the problem is computationally harder than deciding membership. The correctness proof of the algorithm yields a stronger result, namely a description of a possible separator. Finally, we discuss the same problem for context-free input languages.
\end{abstract}

\section{INTRODUCTION}

Context. The strong connection between finite state devices and descriptive formalisms, such as first-order or monadic second-order logic, has been a guideline in computer science since the seminal work of Büchi [7], Elgot [14] and Trakhtenbrot [45]. This bridge has continuously been fruitful, disseminating tools and bringing a number of applications outside of its original research area. For instance, compiling logical specifications into various forms of automata has become one of the most successful methods in automatic program verification [46].

One of the challenging issues when dealing with a logical formalism is to precisely understand its expressiveness and its limitations. While solutions to decide such logics often use a compilation procedure from formulas to automata, capturing the expressive power amounts to the opposite translation: given a language, one wants to know whether one can reconstruct a formula that describes it. In other words, we want to solve an instance of the membership problem, which asks whether an input language belongs to some given class.

For regular languages of finite words, the main tool developed to capture this expressive power is the syntactic monoid [29]: this is a finite, computable, algebraic abstraction of the

2012 ACM CCS: [Theory of computation]: Formal languages and automata theory - Regular languages.

Key words and phrases: Automata, Logics, Monoids, Locally testable, Locally Threshold Testable, Separation, Context-free Language.

Supported by the Agence Nationale de la Recherche ANR 2010 BLAN 020201 FREC.

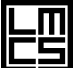
IN COMPUTER SCIENCE
DOI:10.2168/LMCS-10(3:24)2014
Thomas Place, Lorijn van Rooijen, and Marc Zeitoun

(c) Creative Commons 
language, whose properties make it possible to decide membership. An emblematic example is the membership problem for the class of first-order definable languages, solved by Schützenberger [36] and McNaughton and Papert [26], which has led to the development of algebraic methods for obtaining decidable characterizations of logical or combinatorial properties.

The separation problem and its motivations. We consider here the separation problem as a generalization of the membership problem. Assume we are given two classes of languages $\mathcal{C}$ and $\mathcal{S}$. The question is, given two input languages from $\mathcal{C}$, whether we can separate them by a language from $\mathcal{S}$. Here, we say that a language separates $K$ from $L$ if it contains $K$ and is disjoint from $L$. An obvious necessary condition for separability is that the input languages $K, L$ be disjoint. A separator language witnesses this condition.

One strong motivation for this problem is to understand the limits of logics over finite words. Notice that membership reduces to separation when $\mathcal{C}$ is closed under complement, because checking that a language belongs to $\mathcal{S}$ amounts to testing that it is $\mathcal{S}$-separable from its complement. Deciding $\mathcal{S}$-separation is also more difficult than deciding membership in $\mathcal{S}$, as one cannot rely on algebraic tools tailored to the membership problem. It may also be computationally harder, as we shall see in this paper. Thus, solving the separation problem requires a deeper understanding of $\mathcal{S}$ than what is sufficient to check membership: one not only wants to decide whether $\mathcal{S}$ is powerful enough to describe a language, but also to decide whether it can discriminate between two input languages. This discriminating power provides more accurate information than the expressive power.

Contributions. In general, elements of $\mathcal{C}$ cannot always be separated by an element of $\mathcal{S}$ and there is no minimal separator wrt. inclusion. We are interested in the following questions:

(a) can we decide whether one can separate two given languages of $\mathcal{C}$ by a language of $\mathcal{S}$ ?

(b) what is the complexity of this decision problem?

(c) if separation is possible, can we compute a separator, and at which cost?

We investigate the separation problem by locally and locally threshold testable languages. A language is called locally testable (LT) if membership of a word can be tested by inspecting its prefixes, suffixes and infixes up to some length (which depends on the language). The membership problem for this class was raised by McNaughton and Papert [26], and solved independently by McNaughton and Zalcstein [49, 25] and by Brzozowski and Simon [6]. If the input language is given by a deterministic automaton, the membership problem to LT is PTIme [20]. This class has several generalizations. The most studied one is that of locally threshold testable languages (LTT), where counting infixes is allowed up to some threshold. These are the languages definable in $\mathrm{FO}(+1)$, i.e., first-order logic with the successor relation (but without the order). Again, membership is decidable [42], and can actually be again tested in Pтіме [28, 30, 44] if the input language is given by a deterministic automaton.

Actually, the decision problem $(a)$ has been rephrased in purely algebraic terms [1]: solving the separation problem for a class $\mathcal{S}$ amounts to computing the so-called 2-pointlike sets for the algebraic variety corresponding to $\mathcal{S}$. It has been shown that both the varieties corresponding to LT and to LTT1 have computable pointlike sets. This is a consequence of [12, 11] for LT and of [2, 40, 38, 39] for LTT. However, this approach suffers some drawbacks.

\footnotetext{
${ }^{1}$ These algebraic varieties are respectively the pseudovariety of semigroups LSI of local semilattices, and the semidirect product Acom $* \mathrm{D}$ of commutative and aperiodic semigroups with right zero semigroups.
} 
- First, the proofs are purely algebraic, and they provide no insight on the underlying class $\mathcal{S}$ of regular languages itself. Instead, the proofs are based on algebraic properties of a profinite semigroup that depends on $\mathcal{S}$, and which is in general uncountable.

- The proofs involve difficult results from profinite semigroup theory, and therefore require a significant background in algebra and topology: this is the second drawback. The separation problem is indeed equivalent to showing that the topological closure of the input languages wrt. the profinite topology do intersect. Deciding nonemptiness of such an intersection in turn requires a deep understanding of the algebraic properties of this profinite semigroup.

- Finally, this approach only provides a yes/no answer, but no description of what an actual separator might be.

The present paper alleviates these drawbacks, by only using elementary pumping arguments, and providing bounds on the parameters defining the separators.

Our results are as follows: we show that separability of regular languages by LT and LTT languages is decidable by reduction to fixed parameters: for a fixed threshold, we provide a bound on the length of infixes that define a possible separator. For LTT-separators, we also provide a bound for a sufficient threshold. This reduces the problem to a finite number of candidate separators, and hence entails decidability. We further get an equivalent formulation on NFAs in terms of forbidden patterns for the languages to be separable, which yields an Nexptime algorithm. We also obtain lower complexity bounds: even starting from DFAs, the problem is NP-hard for LT and LTT (while membership is in PTIME). Finally, we discuss the separation problem starting from context-free input languages rather than regular ones.

The main arguments rely on pumping in monoids or automata. The core of our proof is generic: we show that if one can find two words, one in each input language, that are close enough wrt. the class of separators, then the languages are not separable. Here, "close enough" is defined in terms of parameters of the input languages, such as the size of input NFAs.

The separation problem in other contexts. While our main concern is theoretical, let us mention some motivating applications where separation occurs as a main ingredient.

In model checking, reachable configurations of a system can be represented by a language. Separating it from the language representing bad configurations proves to be effective for verifying safety of a system. Craig interpolation is a form of separation used in this context, as well as in type inference, theorem proving, hardware specification [24, 18. In the same line of thought, separation is the core idea of the reachability algorithm for vector addition systems designed by Leroux [21, who greatly simplified the original decidability proof [23] thanks to a difficult separation theorem: he proved that a recursively enumerable set of separators (namely Presburger definable sets of configurations) witnesses non-reachability. A related problem addressed also in automatic verification is to find separators in terms of small or minimal DFAs [16] using learning algorithms [8, 22. Finally, questions in database theory also motivated separation questions [13]. These applications justify a systematic study of the separation problem. It is therefore surprising that it deserved only little attention and isolated work [9, 10, 41, 19], even in the restricted, yet still challenging case of regular languages.

Related work for other classes. The separation problem has recently been shown to be PTIME-decidable for the class of piecewise-testable languages, independently and with 
different techniques in [13] and [32]. In the latter paper, it has also been solved using elementary pumping arguments for the class of unambiguous languages, a widely studied class that corresponds to languages definable in first-order logic with only 2 variables. The case of full first-order logic, already shown decidable in [17], has been reproved in [34] with the proof canvas of the present paper, which extends to separating languages of infinite words. Recently, the problem has also been shown decidable for separation by $\Sigma_{2}$-definable languages (that is, languages definable by first-order formulas having only 2 alternations and beginning with an existential block). This information has been in turn used to get decidable characterizations for higher levels in the first-order quantifier alternation hierarchy [33].

Paper Outline. We present the necessary background and notation in Section 2, The classes LT and LTT are defined in Section 3. In Section 4, we present our results for solving separation when the counting threshold is assumed to be fixed. This solves separation in particular for the class LT. In Section 5, we show that separation by LTT languages can be decided by bounding the counting threshold. We also provide an optimality result for the bound we obtain. In Section 6, we show upper and lower bounds for the separation problem by LT and LTT languages. In Section 7 , we consider separation by LT and LTT languages when the input languages to be separated are context-free, rather than regular. We finally present some open problems and further work in Section 8

\section{PRELIMINARIES}

Words and Languages. We fix a finite alphabet $A$. We denote by $A^{*}$ the free monoid over $A$. The empty word is denoted by $\varepsilon$. If $w$ is a word, we set $|w|$ as the length, or size of $w$. When $w$ is nonempty, we view $w$ as a sequence of $|w|$ positions labeled over $A$. We number positions from 0 (for the leftmost one) to $|w|-1$ (for the rightmost one).

Infixes, Prefixes, Suffixes. An infix of a word $w$ is a word $w^{\prime}$ such that $w=u \cdot w^{\prime} \cdot v$ for some $u, v \in A^{*}$. Moreover, if $u=\varepsilon$ (resp. $v=\varepsilon$ ) we say that $w^{\prime}$ is a prefix (resp. suffix) of $w$.

Let $0 \leqslant x<y \leqslant|w|$. We write $w[x, y]$ for the infix of $w$ starting at position $x$ and ending at position $y-1$. By convention, we also set $w[x, x]=\varepsilon$. Observe that by definition, when $x \leqslant y \leqslant z$, we have $w[x, z]=w[x, y] \cdot w[y, z]$.

Profiles. For $k \in \mathbb{N}$, let $k_{\ell}=\lfloor k / 2\rfloor$ and $k_{r}=k-k_{\ell}$. A $k$-profile is a pair of words $\left(w_{\ell}, w_{r}\right)$ of lengths at most $k_{\ell}$ and $k_{r}$, respectively. Given $w \in A^{*}$ and $x$ a position of $w$, the $k$-profile of $x$ is the pair $\left(w_{\ell}, w_{r}\right)$ defined as follows: $w_{\ell}=w\left[\max \left(0, x-k_{\ell}\right), x\right]$ and $w_{r}=w\left[x, \min \left(x+k_{r},|w|\right)\right]$ (see Figure 1). A $k$-profile $\left(w_{\ell}, w_{r}\right)$ occurs in a word $w$ if there exists some position $x$ within $w$ whose $k$-profile is $\left(w_{\ell}, w_{r}\right)$. Similarly, if $n$ is a natural number, we say that $\left(w_{\ell}, w_{r}\right)$ occurs $n$ times in $w$ if there are $n$ distinct positions in $w$ where $\left(w_{\ell}, w_{r}\right)$ occurs. We denote by $A_{k}$ the set of $k$-profiles over $A$. Note that its size is $\left|A_{k}\right|=|A|^{O(k)}$.

Intuitively, the $k$-profile is the description of the infix of $w$ that is centered at position $x$. Observe in particular that the $k$-profiles that occur in a word determine the prefixes and suffixes of length $(k-1)$ of this word. This is convenient, since we only have to consider one object instead of three as in the usual presentations of the classes LT and LTT.

Separability. Given languages $L, L_{1}, L_{2}$ over $A^{*}$, we say that $L$ separates $L_{1}$ from $L_{2}$ if

$$
L_{1} \subseteq L \text { and } L_{2} \cap L=\varnothing .
$$




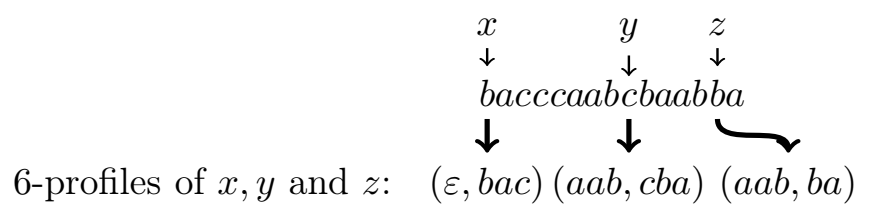

Figure 1: Illustration of the notion of $k$-profile for $k=6$

Given a class $\mathcal{S}$ of languages, we say that the pair $\left(L_{1}, L_{2}\right)$ is $\mathcal{S}$-separable if some language $L \in \mathcal{S}$ separates $L_{1}$ from $L_{2}$. When $\mathcal{S}$ is closed under complement, $\left(L_{1}, L_{2}\right)$ is $\mathcal{S}$-separable if and only if $\left(L_{2}, L_{1}\right)$ is, in which case we simply say that $L_{1}$ and $L_{2}$ are $\mathcal{S}$-separable.

Automata. A nondeterministic finite automaton (NFA) over $A$ is denoted by a tuple $\mathcal{A}=(Q, A, \delta)$, where $Q$ is the finite set of states and $\delta \subseteq Q \times A \times Q$ is the transition relation. Observe that this definition is not the standard one: we do not include the initial and final state sets (we shall explain below why this is more convenient in our setting). Abusing notation, we also denote by $\delta \subseteq Q \times A^{*} \times Q$ the relation induced by the transition relation. Given sets $I \subseteq Q$ and $F \subseteq Q$, we then set

$$
L(\mathcal{A}, I, F)=\left\{w \in A^{*} \mid \exists q_{I} \in I, \exists q_{F} \in F,\left(q_{I}, w, q_{F}\right) \in \delta\right\} .
$$

A language $L \subseteq A^{*}$ is accepted, or recognized by $\mathcal{A}$ if there exist sets $I \subseteq Q$ and $F \subseteq Q$ such that $L=L(\mathcal{A}, I, F)$. In this case, we call $I$ the set of initial states and $F$ the set of final states for $L$. The reason why we do not include initial and final states in our definition of an NFA is the following: when dealing with separation, we start from two input languages. However, it is convenient to work with a single automaton recognizing them both, rather than having to deal with two. Our definition permits this: an NFA recognizing both languages can be obtained by building the Cartesian product of NFAs recognizing the input languages, and by then suitably choosing initial and final states for each of the input languages.

The size $|\mathcal{A}|$ of an automaton $\mathcal{A}$ is its number of states plus its number of transitions. If $\delta$ is a function, then $\mathcal{A}$ is called a deterministic finite automaton (DFA).

Monoids. Let $L$ be a language and let $M$ be a monoid. We say that $L$ is recognized by $M$ if there exists a monoid morphism $\alpha: A^{*} \rightarrow M$ together with a subset $F \subseteq M$ such that $L=\alpha^{-1}(F)$. We also say in this case that $L$ is recognized by $\alpha$. It is well known that a language is accepted by an NFA if and only if it can be recognized by a finite monoid. We denote by $|M|$ the size of a finite monoid.

In the same way as for NFAs, we want to work with a single monoid recognizing both input languages. This is easy to obtain, since if $\alpha_{1}: A^{*} \rightarrow M_{1}$ (resp. $\alpha_{2}: A^{*} \rightarrow M_{2}$ ) recognizes $L_{1}=\alpha_{1}^{-1}\left(F_{1}\right)$ (resp. $L_{2}=\alpha_{2}^{-1}\left(F_{2}\right)$ ), then $\alpha: A^{*} \rightarrow M_{1} \times M_{2}$ defined by $\alpha(w)=\left(\alpha_{1}(w), \alpha_{2}(w)\right)$ recognizes both $L_{1}=\alpha^{-1}\left(F_{1} \times M_{2}\right)$ and $L_{2}=\alpha^{-1}\left(M_{1} \times F_{2}\right)$.

Finally, it is well known that one can compute from any NFA a finite monoid recognizing any language that this NFA can accept. The easiest way to do so is to consider the transition monoid of the NFA, which is generated by Boolean matrices $M_{a} \in\{0,1\}^{Q \times Q}$ for $a \in A$, where $M_{a}(p, q)=1$ if $(p, a, q) \in \delta$ and $M_{a}(p, q)=0$ otherwise. It is straightforward to check that this finite monoid recognizes any language accepted by $\mathcal{A}$. 


\section{Locally Testable and Locally Threshold Testable Languages}

In this paper, we investigate two classes of languages. Intuitively, a language is locally testable if membership of a word in the language only depends on the set of infixes, prefixes and suffixes up to some fixed length that occur in the word. For a locally threshold testable language, membership may also depend on the number of occurrences of such infixes, which may thus be counted up to some fixed threshold.

In this section we provide specific definitions for both classes. We start with the larger class of locally threshold testable languages. In the following, we say that two numbers are equal up to threshold $d$ if either both numbers are equal, or both are greater than or equal to $d$.

Locally Threshold Testable Languages. We say that a language is locally threshold testable (LTT) if it is a boolean combination of languages of the form:

(1) $u A^{*}=\{w \mid u$ is a prefix of $w\}$, for some $u \in A^{*}$.

(2) $A^{*} u=\{w \mid u$ is a suffix of $w\}$, for some $u \in A^{*}$.

(3) $\{w \mid w$ has $u$ as an infix at least $d$ times $\}$, for some $u \in A^{*}$ and $d \in \mathbb{N}$.

Actually, LTT languages can be defined in terms of first-order logic: a language is LTT if and only if it can be defined by an $\mathrm{FO}(+1)$ formula, i.e., a first-order logic formula using predicates for the equality and next position relations, but not for the linear order. See [2, 43].

We also define an index on LTT languages. Usually, this index is defined as the smallest size of infixes, prefixes and suffixes needed to define the language. However, since we only work with $k$-profiles, we directly define an index based on the size of $k$-profiles. Given a $k$-profile $\left(w_{\ell}, w_{r}\right)$, let $\mid w_{\left(w_{\ell}, w_{r}\right)}$ be the number of positions $x$ in $w$ such that $\left(w_{\ell}, w_{r}\right)$ is the $k$-profile of $x$. For $w, w^{\prime} \in A^{*}$ and $k, d \in \mathbb{N}$, we write $w \equiv_{k}^{d} w^{\prime}$ if for every $k$-profile $\left(w_{\ell}, w_{r}\right)$, the numbers $|w|_{\left(w_{\ell}, w_{r}\right)}$ and $\left|w^{\prime}\right|_{\left(w_{\ell}, w_{r}\right)}$ are equal up to threshold $d$.

One can verify that $\equiv_{k}^{d}$ is an equivalence relation (and actually a congruence) of finite index. For $k, d \in \mathbb{N}$, let us denote by $\operatorname{LTT}[k, d]$ the set of the finitely many languages that are unions of $\equiv_{k}^{d}$-classes. By definition, we have LTT $=\bigcup_{k, d} \operatorname{LTT}[k, d]$. Given $L \subseteq A^{*}$, the smallest $\operatorname{LTT}[k, d]$-language containing $L$ is

$$
[L]_{k}^{d}=\left\{w \in A^{*} \mid \exists u \in L \text { such that } u \equiv_{k}^{d} w\right\} .
$$

As it is often the case, there is no smallest LTT language containing a given regular language. For instance, over $A=\{a\}$, any LTT language containing $(a a)^{*}$ is of the form $a^{2 p} a^{*} \cup$ $F$ with $F$ finite. Removing $a^{2 p+1}$ from such a language yields a smaller LTT one, still containing $(a a)^{*}$.

Locally Testable Languages. The class of locally testable languages is the restriction of LTT languages in which infixes cannot be counted. A language is locally testable (LT) if it is a boolean combination of languages of the form (11), (2) and the following restriction of (3):

(4) $A^{*} u A^{*}=\{w \mid w$ has $u$ as an infix $\}$, for some $u \in A^{*}$.

No simple description of LT in terms of first-order logic is known. In terms of linear temporal logic, LT languages are exactly those defined by formulas involving only the operators $\mathbf{F}$ (eventually) and $\mathbf{X}$ (next), with no nesting of $\mathbf{F}$ operators.

Given two words $w, w^{\prime}$ and a number $k$, we write $w \equiv_{k} w^{\prime}$ for $w \equiv_{k}^{1} w^{\prime}$. For all $k \in \mathbb{N}$, we denote by $\mathrm{LT}[k]$ the set of languages that are unions of $\equiv_{k}$-classes, and LT $=\bigcup_{k} \mathrm{LT}[k]$. 
Given $L \subseteq A^{*}$ and $k \in \mathbb{N}$, the smallest $\mathrm{LT}[k]$-language containing $L$ is

$$
[L]_{k}=\left\{w \in A^{*} \mid \exists u \in L \text { such that } u \equiv_{k} w\right\} .
$$

However, as for LTT, there is no smallest LT language containing a given regular language.

\section{Separation for a Fixed Threshold}

In this section, we prove that if $d$ is a fixed natural number, it is decidable whether two languages can be separated by an LTT language of counting threshold $d$ (i.e., by an LTT $[k, d]$ language for some $k$ ). In particular, this covers the case of LT, which corresponds to $d=1$. All results in this section are for an arbitrary fixed $d$. Our result is twofold.

- First, we establish a bound $k$ on the size of profiles that it suffices to consider in order to separate the input languages. This bound only depends on the size of monoids recognizing these languages, and it can be computed. One can then use a brute-force algorithm that tests separability by all the finitely many $\operatorname{LTT}[k, d]$ languages.

- The second contribution is a criterion on the input languages to check separability by an LTT $[k, d]$ language for some $k$. This criterion can be defined equivalently on automata or monoids recognizing the input languages, in terms of the absence of common patterns.

The section is divided in four subsections. Our criterion is stated in the first one. The remaining subsections are then devoted to the statement and proof of the theorem.

4.1. Patterns. In this section, we define our criterion that two languages must satisfy in order to be separable. The criterion can be defined equivalently on automata or monoids recognizing the languages.

Block Patterns. A block is a triple of words $\mathfrak{b}=\left(v_{\ell}, u, v_{r}\right)$ where $v_{\ell}, v_{r}$ are nonempty. Similarly, a prefix block is a pair of words $\mathfrak{p}=\left(u, v_{r}\right)$ with $v_{r}$ nonempty, and a suffix block is a pair of words $\mathfrak{s}=\left(v_{\ell}, u\right)$ with $v_{\ell}$ nonempty. Let $d \in \mathbb{N}$. A d-pattern $\mathcal{P}$ is

- either a word $w$,

- or a triple $(\mathfrak{p}, f, \mathfrak{s})$ where $\mathfrak{p}$ and $\mathfrak{s}$ are respectively a prefix and a suffix block, and $f$ is a function mapping blocks to the set $\{0, \ldots, d\}$, such that all but finitely many blocks are mapped to 0 .

Decompositions. Let $w$ be a word and let $\mathcal{P}$ be a $d$-pattern. We say that $w$ admits a $\mathcal{P}$-decomposition if $w$ admits a decomposition $w=u_{0} v_{1} u_{1} v_{2} \cdots v_{n} u_{n}$ with $n \geqslant 0$ and such that either $n=0$ and $\mathcal{P}=u_{0}=w$, or $\mathcal{P}=(\mathfrak{p}, f, \mathfrak{s})$ and the following conditions are verified:

(1) $\mathfrak{p}=\left(u_{0}, v_{1}\right)$ and $\mathfrak{s}=\left(v_{n}, u_{n}\right)$.

(2) for every block $\mathfrak{b}$, if $f(\mathfrak{b})<d$, then $\left|\left\{i \mid\left(v_{i}, u_{i}, v_{i+1}\right)=\mathfrak{b}\right\}\right|=f(\mathfrak{b})$.

(3) for every block $\mathfrak{b}$, if $f(\mathfrak{b})=d$, then $\left|\left\{i \mid\left(v_{i}, u_{i}, v_{i+1}\right)=\mathfrak{b}\right\}\right| \geqslant d$.

We may say $\mathcal{P}$-decomposition to mean $\mathcal{P}$-decomposition of some word. Let $\alpha: A^{*} \rightarrow M$ be a morphism into a monoid $M$, and let $s \in M$. A $\mathcal{P}$-decomposition of $w$ is said to be $(\alpha, s)$ compatible if $\alpha(w)=s$ and $\alpha\left(u_{0} \cdots v_{i}\right)=\alpha\left(u_{0} \cdots v_{i}\right) \cdot \alpha\left(v_{i}\right)$, for all $1 \leqslant i \leqslant n$. Similarly, if $p, q$ are two states of an automaton $\mathcal{A}$, a $\mathcal{P}$-decomposition is $(p, q)$-compatible if there is a run from $p$ to $q$ on $w$, such that for all $1 \leqslant i \leqslant n$, each infix $v_{i}$ labels a loop in the run, as pictured in Figure 2 


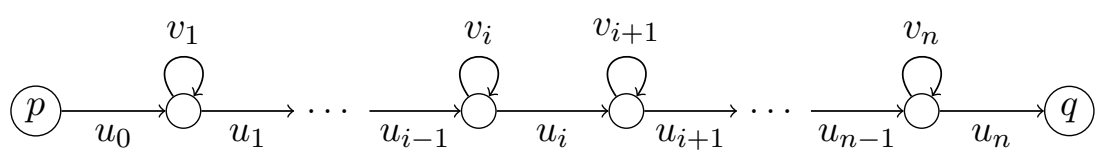

Figure 2: A $(p, q)$-compatible $\mathcal{P}$-decomposition $u_{0} v_{1} \cdots v_{n} u_{n}$ (edges denote transition sequences)

Common Patterns. Let $d \in \mathbb{N}$ and $\alpha: A^{*} \rightarrow M$ be a morphism into a finite monoid. We say that a pair $\left(s_{1}, s_{2}\right) \in M \times M$ has a common d-pattern if there exist a $d$-pattern $\mathcal{P}$ and two $\mathcal{P}$-decompositions of (possibly different) words that are respectively $\left(\alpha, s_{1}\right)$-compatible and $\left(\alpha, s_{2}\right)$-compatible. In this terminology, $\alpha$ is understood and not mentioned explicitly. Similarly, if $\mathcal{A}$ is an automaton, and $p_{1}, q_{1}, p_{2}, q_{2}$ are states of $\mathcal{A}$, we say that the pair $\left(\left(p_{1}, q_{1}\right),\left(p_{2}, q_{2}\right)\right)$ has a common d-pattern if there exist a $d$-pattern $\mathcal{P}$ and two $\mathcal{P}$ decompositions of words that are respectively $\left(p_{1}, q_{1}\right)$-compatible and $\left(p_{2}, q_{2}\right)$-compatible. In particular, $\left(\left(p_{1}, q_{1}\right),\left(p_{2}, q_{2}\right)\right)$ has a common 1-pattern if there are paths in $\mathcal{A}$ of the form shown in Figure 2 with the same set of triples $\left(v_{i}, u_{i}, v_{i+1}\right)$, going respectively from $p_{1}$ to $q_{1}$ and from $p_{2}$ to $q_{2}$.

4.2. Separation Theorem for a Fixed Threshold. The reason for introducing common patterns is the following. First, having a common $d$-pattern for a pair $\left(s_{1}, s_{2}\right) \in M \times M$ is a decidable property. Second, it is a necessary and sufficient condition for the languages $\alpha^{-1}\left(s_{1}\right)$ and $\alpha^{-1}\left(s_{2}\right)$ not being separable by any $\operatorname{LTT}[k, d]$ language, for any $k$. A similar statement holds for common $d$-patterns in NFAs. This is what we state now in our main theorem for this section.

Theorem 4.1. Fix $d \in \mathbb{N}$. Let $L_{1}, L_{2}$ be regular languages. Let $\alpha: A^{*} \rightarrow M$ be a morphism into a finite monoid $M$ recognizing both $L_{1}$ and $L_{2}$. Let $\mathcal{A}$ be an NFA recognizing both $L_{1}$ and $L_{2}$, with $L_{i}=L\left(\mathcal{A}, I_{i}, F_{i}\right)$. Set $k=4(|M|+1)$. Then, the following conditions are equivalent:

(1) $L_{1}$ and $L_{2}$ are $\operatorname{LTT}[\ell, d]$-separable for some $\ell$.

(2) $L_{1}$ and $L_{2}$ are LTT $[k, d]$-separable.

(3) The language $\left[L_{1}\right]_{k}^{d}$ separates $L_{1}$ from $L_{2}$.

(4) No pair in $\alpha\left(L_{1}\right) \times \alpha\left(L_{2}\right)$ has a common d-pattern.

(5) No pair in $\left(I_{1} \times F_{1}\right) \times\left(I_{2} \times F_{2}\right)$ has a common d-pattern.

Observe that Item (2) is essentially a delay theorem [40] for separation restricted to the case of LTT: we prove that the size of profiles (i.e., infixes) that a potential separator needs to consider can be bounded by a function of the size of the monoids recognizing the languages. By restricting Theorem 4.1 to the case $d=1$, we get the following separation theorem for LT, that we explicitly state in view of the relevance of this class.

Theorem 4.2. Let $L_{1}, L_{2}$ be regular languages. Let $\alpha: A^{*} \rightarrow M$ be a morphism into a finite monoid $M$ recognizing both $L_{1}$ and $L_{2}$. Let $\mathcal{A}$ be an $N F A$ recognizing both $L_{1}$ and $L_{2}$, with $L_{i}=L\left(\mathcal{A}, I_{i}, F_{i}\right)$. Set $k=4(|M|+1)$. The following conditions are equivalent:

(1) $L_{1}$ and $L_{2}$ are LT-separable.

(2) $L_{1}$ and $L_{2}$ are $\mathrm{LT}[k]$-separable.

(3) The language $\left[L_{1}\right]_{k}$ separates $L_{1}$ from $L_{2}$. 
(4) No pair in $\alpha\left(L_{1}\right) \times \alpha\left(L_{2}\right)$ has a common 1-pattern.

(5) No pair in $\left(I_{1} \times F_{1}\right) \times\left(I_{2} \times F_{2}\right)$ has a common 1-pattern.

Theorem4.1 and Theorem 4.2 yield algorithms for deciding LTT- and LT-separability for a fixed threshold. Indeed, the algorithm just tests all the finitely many LTT $[k, d]$ languages as potential separators. This brute-force approach yields a very costly procedure. It turns out that a more practical algorithm can be obtained from Items (4) and (5). We postpone the presentation of this algorithm to Section 6 .

Corollary 4.3. Let $d \in \mathbb{N}$. It is decidable whether two given regular languages are LTT $[\ell, d]$ separable for some $\ell \in \mathbb{N}$. In particular, it is decidable whether they are LT-separable.

It remains to prove Theorem 4.1. The implications (31) $\Rightarrow$ (2) $\Rightarrow$ (11) are immediate by definition. We now prove the implications (10) $\Rightarrow(15) \Rightarrow(44) \Rightarrow(\underline{3})$, devoting a separate subsection to the proof of each implication.

4.3. Implication (11) $\Rightarrow$ (5) in Theorem 4.1. We prove the contrapositive of (11) $\Rightarrow$ (5): if $\left(\left(p_{1}, q_{1}\right),\left(p_{2}, q_{2}\right)\right) \in\left(I_{1} \times F_{1}\right) \times\left(I_{2} \times F_{2}\right)$ has a common $d$-pattern, then there exists no $\ell \in \mathbb{N}$ such that $L_{1}$ and $L_{2}$ are LTT $[\ell, d]$-separable. This is an immediate consequence of the next proposition.

Proposition 4.4. Fix $d \in \mathbb{N}$ and let $\mathcal{A}$ be an NFA. Let $p_{1}, q_{1}, p_{2}, q_{2}$ be states of $\mathcal{A}$. If $\left(\left(p_{1}, q_{1}\right),\left(p_{2}, q_{2}\right)\right)$ has a common d-pattern, then, for all $\ell \in \mathbb{N}$, there exist $w_{1}, w_{2}$ accepted respectively by $L\left(\mathcal{A},\left\{p_{1}\right\},\left\{q_{1}\right\}\right)$ and $L\left(\mathcal{A},\left\{p_{2}\right\},\left\{q_{2}\right\}\right)$ such that $w_{1} \equiv_{\ell}^{d} w_{2}$.

Proof. Set $L_{1}=L\left(\mathcal{A},\left\{p_{1}\right\},\left\{q_{1}\right\}\right)$ and $L_{2}=L\left(\mathcal{A},\left\{p_{2}\right\},\left\{q_{2}\right\}\right)$. Let $\mathcal{P}$ be a common $d$-pattern of $\left(\left(p_{1}, q_{1}\right),\left(p_{2}, q_{2}\right)\right)$. If $\mathcal{P}=w \in A^{*}$, then by definition, $w \in L_{1} \cap L_{2}$, and it suffices to choose $w_{1}=w_{2}=w$. Otherwise, $\mathcal{P}=(\mathfrak{p}, f, \mathfrak{s})$ and there exist $z_{1} \in L_{1}, z_{2} \in L_{2}$ having a $\left(p_{1}, q_{1}\right)$ - respectively $\left(p_{2}, q_{2}\right)$-compatible $\mathcal{P}$-decomposition. Let $z_{1}=u_{0} v_{1} u_{1} v_{2} \cdots v_{n} u_{n}$ and $z_{2}=x_{0} y_{1} x_{1} y_{2} \cdots y_{m} x_{m}$ be these decompositions. For $\ell \in \mathbb{N}$, set

$$
\begin{aligned}
& w_{1}=u_{0} v_{1}^{\ell(d+1)} u_{1} v_{2}^{\ell(d+1)} \cdots v_{n}^{\ell(d+1)} u_{n}, \\
& w_{2}=x_{0} y_{1}^{\ell(d+1)} x_{1} y_{2}^{\ell(d+1)} \cdots y_{m}^{\ell(d+1)} x_{m} .
\end{aligned}
$$

By definition of compatibility, we deduce that $w_{1} \in L_{1}$ and $w_{2} \in L_{2}$. We claim that $w_{1} \equiv_{\ell}^{d} w_{2}$. Indeed, from the $\mathcal{P}$-decompositions of $z_{1}$ and $z_{2}$, we deduce that $u_{0}=x_{0}$ and $v_{1}=y_{1}$, which implies that $w_{1}$ and $w_{2}$ have the same prefix of length $\ell-1$ (and actually, even of length $\ell(d+1)$ ). Similarly, they have the same suffix of length $\ell-1$. To show the claim, it remains to verify that each word of length at most $\ell$ occurs the same number of times, up to threshold $d$, as an infix in $w_{1}$ and in $w_{2}$. Let $u$ be an infix of length at most $\ell$ of, say, $w_{1}$.

Assume first that $u$ occurs in some $v_{i}^{\ell(d+1)}$. Then it occurs at least $d$ times. Since the decompositions of $w_{1}, w_{2}$ are $\mathcal{P}$-decompositions, there exists some $j$ such that $y_{j}=v_{i}$. Therefore, $u$ occurs at least $d$ times as an infix in $y_{j}^{\ell(d+1)}$, hence also in $w_{2}$.

Assume finally that $u$ does not occur in any $v_{i}^{\ell(d+1)}$. Therefore, it overlaps with some of the $u_{i}$ 's, and for these indices $i$, it is an infix of $v_{i}^{\ell(d+1)} u_{i} v_{i+1}^{\ell(d+1)}$. Since both decompositions of $w_{1}$ and $w_{2}$ are $\mathcal{P}$-compatible, the number of triples $\left(v_{i}, u_{i}, v_{i+1}\right)$ in the decomposition of $w_{1}$ and the number of triples $\left(y_{j}, x_{j}, y_{j+1}\right)$ in that of $w_{2}$ which are equal to a given triple is the same, up to threshold $d$. Therefore, $u$ occurs the same number of times up to threshold $d$ in both $w_{1}$ and $w_{2}$. We have thus shown that $w_{1} \equiv_{\ell}^{d} w_{2}$. 
4.4. Implication (5) $\Rightarrow$ (4) in Theorem 4.1. We prove the contrapositive: if there is a pair $\left(s_{1}, s_{2}\right) \in \alpha\left(L_{1}\right) \times \alpha\left(L_{2}\right)$ having a common $d$-pattern, then there is a pair $\left(\left(p_{1}, q_{1}\right),\left(p_{2}, q_{2}\right)\right) \in$ $\left(I_{1} \times F_{1}\right) \times\left(I_{2} \times F_{2}\right)$ also having a common $d$-pattern. This follows from the following claim, which states that the presence of a "recognizing pair" $\left(s_{1}, s_{2}\right)$ that has a common $d$-pattern does not depend on the choice of the recognizing monoid morphisms.

Claim. Let $d \in \mathbb{N}$, and let $\alpha: A^{*} \rightarrow M$ and $\beta: A^{*} \rightarrow N$ be monoid morphisms recognizing both $L_{1}$ and $L_{2}$. If there exists $\left(s_{1}, s_{2}\right) \in \alpha\left(L_{1}\right) \times \alpha\left(L_{2}\right)$ having a common d-pattern, then there exists $\left(t_{1}, t_{2}\right) \in \beta\left(L_{1}\right) \times \beta\left(L_{2}\right)$ also having a common d-pattern.

Let us admit the claim for a moment. Assume that $\left(s_{1}, s_{2}\right) \in \alpha\left(L_{1}\right) \times \alpha\left(L_{2}\right)$ has a common $d$-pattern. Let $N$ be the transition monoid of $\mathcal{A}$, and let $\beta: A^{*} \rightarrow N$ be the associated morphism. Since $\beta$ recognizes $L_{i}$ (see [29]), one can apply the claim: it follows that there exists $\left(t_{1}, t_{2}\right) \in \beta\left(L_{1}\right) \times \beta\left(L_{2}\right)$ having a common $d$-pattern. By definition of a transition monoid, it is then immediate to build from $\left(t_{1}, t_{2}\right)$ a pair $\left(\left(p_{1}, q_{1}\right),\left(p_{2}, q_{2}\right)\right) \in$ $\left(I_{1} \times F_{1}\right) \times\left(I_{2} \times F_{2}\right)$ having a common $d$-pattern.

It remains to prove the claim. Let $\alpha: A^{*} \rightarrow M$ and $\beta: A^{*} \rightarrow N$ be morphisms recognizing both $L_{i}$ for $i=1,2$, and let $F_{i}=\alpha\left(L_{i}\right)$ and $G_{i}=\beta\left(L_{i}\right)$. Let $\mathcal{P}$ be a common $d$-pattern of $\left(s_{1}, s_{2}\right) \in F_{1} \times F_{2}$. If $\mathcal{P}=w \in A^{*}$, then by definition $w \in \alpha^{-1}\left(F_{1}\right) \cap \alpha^{-1}\left(F_{2}\right)=$ $L_{1} \cap L_{2}=\alpha^{-1}\left(G_{1}\right) \cap \alpha^{-1}\left(G_{2}\right)$, so $\mathcal{P}$ is a common $d$-pattern of $(\beta(w), \beta(w)) \in G_{1} \times G_{2}$. Otherwise, $\mathcal{P}$ is of the form $(\mathfrak{p}, f, \mathfrak{s})$. We define a new $d$-pattern $\mathcal{P}^{\prime}$ that is common to some $\left(t_{1}, t_{2}\right) \in G_{1} \times G_{2}$. Let $\omega \in \mathbb{N}$ be such that $s^{\omega}$ is idempotent for all $s \in M$ and $s \in N$. For any block $\mathfrak{b}=\left(v_{\ell}, u, v_{r}\right)$, set $\tilde{\mathfrak{b}}=\left(\left(v_{\ell}\right)^{\omega}, u\left(v_{r}\right)^{\omega},\left(v_{r}\right)^{\omega}\right)$. The mapping $\mathfrak{b} \mapsto \tilde{\mathfrak{b}}$ is clearly injective. Set $\mathcal{P}^{\prime}$ as the $d$-pattern $\left(\mathfrak{p}^{\prime}, f^{\prime}, \mathfrak{s}^{\prime}\right)$ defined as follows:

- $\mathfrak{p}^{\prime}=\left(u\left(v_{r}\right)^{\omega},\left(v_{r}\right)^{\omega}\right)$ with $\left(u, v_{r}\right)=\mathfrak{p}$.

- For any block $\mathfrak{b}^{\prime}$, if $\mathfrak{b}^{\prime}=\tilde{\mathfrak{b}}$ for some block $\mathfrak{b}$, then $f^{\prime}\left(\mathfrak{b}^{\prime}\right)=f(\mathfrak{b})$. Otherwise $f^{\prime}\left(\mathfrak{b}^{\prime}\right)=0$.

- $\mathfrak{s}^{\prime}=\left(\left(v_{\ell}\right)^{\omega}, u\right)$ with $\left(v_{\ell}, u\right)=\mathfrak{s}$.

We now prove that $\mathcal{P}^{\prime}$ is a common $d$-pattern of some $\left(t_{1}, t_{2}\right) \in G_{1} \times G_{2}$. By definition of $\mathcal{P}$, there exist $z_{1} \in \alpha^{-1}\left(s_{1}\right) \subseteq L_{1}, z_{2} \in \alpha^{-1}\left(s_{2}\right) \subseteq L_{2}$ having an $\left(\alpha, s_{1}\right)$-, respectively $\left(\alpha, s_{2}\right)$ compatible $\mathcal{P}$-decomposition. Let $z_{1}=u_{0} v_{1} u_{1} v_{2} \cdots v_{n} u_{n}$ and $z_{2}=x_{0} y_{1} x_{1} y_{2} \cdots y_{m} x_{m}$ be these decompositions. Set $\tilde{u}_{i}=u_{i} v_{i+1}^{\omega}$ for $i<n, \tilde{x}_{i}=x_{i} y_{i+1}^{\omega}$ for $i<m, \tilde{u}_{n}=u_{n}, \tilde{x}_{m}=x_{m}$, $\tilde{v}_{i}=v_{i}^{\omega}$ for $i \leqslant n$, and $\tilde{y}_{i}=y_{i}^{\omega}$ for $i \leqslant m$. Let then

$$
\begin{aligned}
& w_{1}=\tilde{u}_{0} \tilde{v}_{1} \tilde{u}_{1} \tilde{v}_{2} \cdots \tilde{v}_{n} \tilde{u}_{n}, \\
& w_{2}=\tilde{x}_{0} \tilde{y}_{1} \tilde{x}_{1} \tilde{y}_{2} \cdots \tilde{y}_{m} \tilde{x}_{m} .
\end{aligned}
$$

It is immediate by definition of $\mathcal{P}^{\prime}$ and $z_{1}, z_{2}$ that $w_{1}, w_{2}$ are $\mathcal{P}^{\prime}$-decompositions. Then, it follows from the definition of $\omega$ for elements of $M$ that $\alpha\left(w_{i}\right) \in F_{i}$ for $i \in\{1,2\}$. Hence, we have $\beta\left(w_{i}\right) \in G_{i}$. We choose $t_{i}=\beta\left(w_{i}\right)$, so that $\left(t_{1}, t_{2}\right) \in G_{1} \times G_{2}$. Finally, it follows from the definition of $\omega$ for $N$ that for $i \in\{1,2\}$, the decomposition of $w_{i}$ given by (4.1) is $\left(\beta, \beta\left(w_{i}\right)\right)$-compatible. We have thus shown that $\left(t_{1}, t_{2}\right) \in G_{1} \times G_{2}$ has a common $d$-pattern.

4.5. Implication (4) $\Rightarrow$ (3) in Theorem 4.1. Again, we prove the contrapositive of the statement: if $\left[L_{1}\right]_{k}^{d}$ does not separate $L_{1}$ from $L_{2}$ when $k=4(|M|+1)$, then there exists $\left(s_{1}, s_{2}\right) \in \alpha\left(L_{1}\right) \times \alpha\left(L_{2}\right)$ having a common $d$-pattern. Observe that by definition of $\left[L_{1}\right]_{k}^{d}$, if $\left[L_{1}\right]_{k}^{d}$ does not separate $L_{1}$ from $L_{2}$, then there exist $w_{1} \in L_{1}, w_{2} \in L_{2}$ with $w_{1} \equiv_{k}^{d} w_{2}$. We 
take $s_{1}=\alpha\left(w_{1}\right)$ and $s_{2}=\alpha\left(w_{2}\right)$. The next proposition shows that the pair $\left(s_{1}, s_{2}\right)$ indeed has a common $d$-pattern.

Proposition 4.5. Let $\alpha: A^{*} \rightarrow M$ be a morphism and let $k=4(|M|+1)$. Let $d \in \mathbb{N}$ and let $w_{1}, w_{2}$ be words such that $w_{1} \equiv_{k}^{d} w_{2}$. Then, there exists a d-pattern $\mathcal{P}$, an $\left(\alpha, \alpha\left(w_{1}\right)\right)$ compatible $\mathcal{P}$-decomposition, and an $\left(\alpha, \alpha\left(w_{2}\right)\right)$-compatible $\mathcal{P}$-decomposition.

The remainder of the section is now devoted to the proof of Proposition 4.5. We set $w_{1}, w_{2}, k$ and $d$ as in the statement of the proposition. Observe first that if $w_{1}=w_{2}=w$, then it suffices to take $\mathcal{P}=w$. Therefore, we suppose for the remainder of the proof that $w_{1} \neq w_{2}$. We proceed as follows: we construct two new words $w_{1}^{\prime}, w_{2}^{\prime}$ from $w_{1}, w_{2}$ such that $w_{1}^{\prime}$ admits an $\left(\alpha, \alpha\left(w_{1}\right)\right)$-compatible $\mathcal{P}$-decomposition and $w_{2}^{\prime}$ admits an $\left(\alpha, \alpha\left(w_{2}\right)\right)$ compatible $\mathcal{P}$-decomposition, for some $d$-pattern $\mathcal{P}=(\mathfrak{p}, f, \mathfrak{s})$.

We first describe the construction of $w_{1}^{\prime}, w_{2}^{\prime}$, and then prove that it is correct. It amounts to duplicating infixes verifying special properties in $w_{1}, w_{2}$. We first define these special infixes, called $k$-loops.

$k$-loops. Let $w \in A^{*}, x$ be a position in $w$, and $\left(w_{\ell}, w_{r}\right)$ be the $\lfloor k / 2\rfloor$-profile of $x$. We say that $x$ admits a $k$-loop if there exists a nonempty prefix $u$ of $w_{r}$ such that $\alpha\left(w_{\ell}\right)=\alpha\left(w_{\ell} \cdot u\right)$. In this case, we call the smallest such $u$ the $k$-loop of $x$. See Figure 3 ,

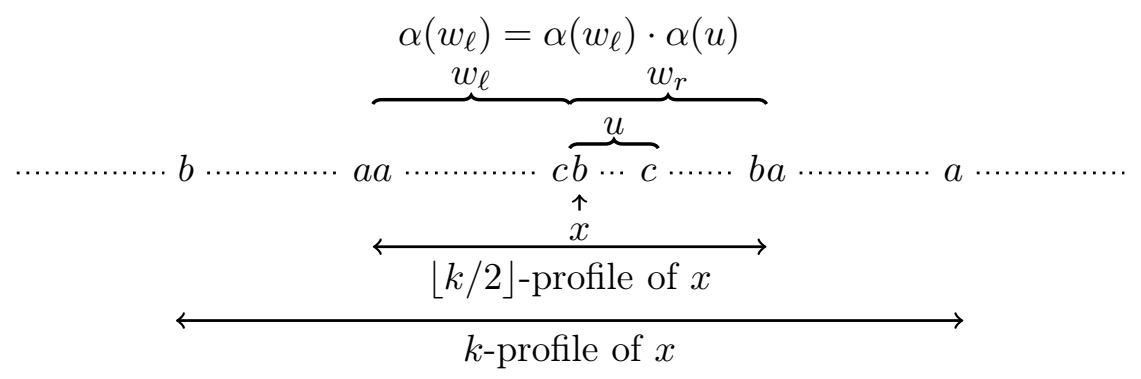

Figure 3: A position $x$ admitting a $k$-loop $u$, that is: $\alpha\left(w_{\ell}\right)=\alpha\left(w_{\ell} \cdot u\right)$

For our construction to work, we need $k$-loops to have three specific properties. The first two are simple facts that are immediate from the definition: $k$-loops are determined by profiles and can be duplicated without modifying the image of the word under $\alpha$.

Fact 4.6. Let $x$ be a position. Whether $x$ admits a $k$-loop, and if so, which $k$-loop $x$ admits, only depends on the $\lfloor k / 2\rfloor$-profile of $x$.

Fact 4.7. Let $w$ be a word and let $x$ be a position within $w$ such that $x$ admits a $k$-loop $u$. Then we have $\alpha(w[0, x])=\alpha(w[0, x]) \cdot \alpha(u)$.

The last property we need is that $k$-loops occur frequently in words, i.e., at least one of $\lfloor k / 4\rfloor$ consecutive positions must admit a $k$-loop. This follows from pumping arguments.

Lemma 4.8. Let $w$ be a word and let $x_{1}, \ldots, x_{\lfloor k / 4\rfloor}$ be $\lfloor k / 4\rfloor$ consecutive positions in $w$. Then, there exists at least one position $x_{i}$ with $i<\lfloor k / 4\rfloor$ that admits a $k$-loop. 
Proof. By choice of $k,\lfloor k / 4\rfloor=|M|+1$. By the pigeonhole principle, we obtain two natural numbers $1 \leqslant i<j \leqslant\lfloor k / 4\rfloor$ such that $\alpha\left(w\left[x_{1}, x_{i}\right]\right)=\alpha\left(w\left[x_{1}, x_{j}\right]\right)$. We prove that $x_{i}$ admits a $k$-loop. Consider the $\lfloor k / 2\rfloor$-profile $\left(w_{\ell}, w_{r}\right)$ of $x_{i}$ and $u=w\left[x_{i}, x_{j}\right]$. Since $\left|w\left[x_{1}, x_{i}\right]\right|<\lfloor k / 4\rfloor$, $w\left[x_{1}, x_{i}\right]$ is a suffix of $w_{\ell}$, so $\alpha\left(w_{\ell}\right)=\alpha\left(w_{\ell} \cdot u\right)$. Since $|u|<\lfloor k / 4\rfloor, u$ is a prefix of $w_{r}$. Therefore $x_{i}$ admits a $k$-loop.

Observe that $u$ is not necessarily the $k$-loop of $x_{i}$, as there might be a smaller word that also satisfies the definition.

Construction of $w_{1}^{\prime}, w_{2}^{\prime}$. We can now construct $w_{1}^{\prime}$ and $w_{2}^{\prime}$. If $w, u$ are words and $x$ is a position of $w$, the word constructed by inserting $u$ at position $x$ is the word $w[0, x] \cdot u \cdot w[x,|w|]$. From $w_{1}$ (resp. $w_{2}$ ), we construct $w_{1}^{\prime}$ (resp. $w_{2}^{\prime}$ ) by inserting simultaneously all infixes $z_{x}$ in $w_{1}$ (resp. $w_{2}$ ) at any position $x$ that admits a $k$-loop, and where $z_{x}$ is the $k$-loop of $x$. It remains to prove that the construction is correct, i.e., that for $i=1,2, w_{i}^{\prime}$ admits an $\left(\alpha, \alpha\left(w_{i}\right)\right)$-compatible $\mathcal{P}$-decomposition for some $d$-pattern $\mathcal{P}=(\mathfrak{p}, f, \mathfrak{s})$.

The Construction is Correct. If we had $\min \left(\left|w_{1}\right|,\left|w_{2}\right|\right) \leqslant\lfloor k / 4\rfloor$, then from $w_{1} \equiv_{k}^{d} w_{2}$ one would obtain $w_{1}=w_{2}$, a case already excluded. Hence, both $w_{1}, w_{2}$ have length at least $\lfloor k / 4\rfloor$, so by Lemma 4.8, at least one insertion has occurred in both $w_{1}$ and $w_{2}$. We now prove that there exists a $d$-pattern $\mathcal{P}=(\mathfrak{p}, f, \mathfrak{s})$ such that for $i=1,2, w_{i}^{\prime}$ admits an $\left(\alpha, \alpha\left(w_{i}\right)\right)$-compatible $\mathcal{P}$-decomposition.

We first define the $d$-pattern $(\mathfrak{p}, f, \mathfrak{s})$. By definition, $w_{1}^{\prime}$ can be decomposed as $w_{1}^{\prime}=$ $u_{0} v_{1} u_{1} v_{2} \cdots v_{n} u_{n}$ with $w_{1}=u_{0} u_{1} \cdots u_{n}$ and the words $v_{j}$ are the $k$-loops inserted in the construction. Since at least one insertion was made, we have $n \geqslant 1$ and we can set $\mathfrak{p}=$ $\left(u_{0}, v_{1}\right), \mathfrak{s}=\left(v_{n}, u_{n}\right)$. We define $f$ as the function that maps a block $\left(v_{\ell}, u, v_{r}\right)$ to the number of times it occurs in the decomposition, up to threshold $d$. Set $\mathcal{P}=(\mathfrak{p}, f, \mathfrak{s})$. By definition, $u_{0} v_{1} u_{1} v_{2} \cdots v_{n} u_{n}$ is a $\mathcal{P}$-decomposition for $w_{1}^{\prime}$. Moreover, it is $\left(\alpha, \alpha\left(w_{1}\right)\right)$-compatible by Fact 4.7. It remains to prove that $w_{2}^{\prime}$ admits an $\left(\alpha, \alpha\left(w_{2}\right)\right)$-compatible $\mathcal{P}$-decomposition.

By definition, $w_{2}^{\prime}$ can be decomposed in a similar way as $w_{1}^{\prime}, w_{2}^{\prime}=u_{0}^{\prime} v_{1}^{\prime} u_{1}^{\prime} v_{2}^{\prime} \cdots v_{m}^{\prime} u_{m}^{\prime}$ with $w_{2}=u_{0}^{\prime} u_{1}^{\prime} \cdots u_{m}^{\prime}$ and the words $v_{j}^{\prime}$ are the $k$-loops inserted in the construction of $w_{2}^{\prime}$. We prove that this is a $\mathcal{P}$-decomposition. It will then be $\left(\alpha, \alpha\left(w_{2}\right)\right)$-compatible by Fact 4.7 ,

To every position $x$ in $w_{2}$ we associate a block, prefix block or suffix block in the following way. By definition, $x$ must fall into a word $u_{i}^{\prime}$ for some $i$. If $i \notin\{0, m\}$, we denote by $g(x)$ the triple $\left(v_{i}^{\prime}, u_{i}^{\prime}, v_{i+1}^{\prime}\right)$. Similarly if $i=0($ resp. $i=m)$, then $g(x)$ is the pair $\left(u_{0}^{\prime}, v_{1}^{\prime}\right)$ (resp. $\left.\left(v_{m}^{\prime}, u_{m}^{\prime}\right)\right)$. The result now follows from the next lemma.

Lemma 4.9. Let $x, y$ be distinct positions of words $w_{1}$ or $w_{2}$ with the same $k$-profile. Then $g(x)=g(y)$. Moreover, the number of copies of a block $\mathfrak{b}$ in the decomposition of $w_{1}$ (resp. $\left.w_{2}\right)$ is exactly the number of positions $x$ in $w_{1}$ (resp. $w_{2}$ ) such that $g(x)=\mathfrak{b}$.

Proof. There are several cases to treat depending on whether $x, y$ are in $w_{1}$ or $w_{2}$ and whether there are at least $\lfloor k / 4\rfloor$ positions to the right and left of $x, y$ or not. All cases are treated similarly. Therefore, we only treat the case when there are at least $\lfloor k / 4\rfloor$ positions to the right and left of $x, y$, and positions $x, y$ are both in $w_{1}$.

Let $z$ be a position such that there are at least $\lfloor k / 4\rfloor$ positions to the right and left of $z$. Let $\left(w_{\ell}, w_{r}\right)$ be the $k$-profile of $z$ and $z_{\ell} \leqslant z$ and $z_{r}>z$ be the positions admitting $k$-loops that are closest to $z$. Observe that by Lemma $4.8, z-z_{\ell} \leqslant\lfloor k / 4\rfloor$ and $z_{r}-z \leqslant\lfloor k / 4\rfloor-1$, hence $z_{\ell}, z_{r}$ belong to the copy of $w_{\ell}, w_{r}$ at $z$.

We claim that the relative positions of $z_{\ell}, z_{r}$ in this copy and the actual $k$-loops only depend on $\left(w_{\ell}, w_{r}\right)$. This claims immediately implies, by construction of $w_{1}^{\prime}, w_{2}^{\prime}$, that $g(z)$ 
only depends on its $k$-profile. This entails the first part of the lemma. Moreover, this also proves that if $\left(v_{\ell}, u, v_{r}\right)=g(z)$, then the relative position of $z$ within the corresponding copy of $u$ only depends on the $k$-profile of $z$. This means that two positions with the same $k$-profile can only generate the same copy of a block if they are equal: this is the second part of the lemma.

It remains to prove the claim. By definition of profiles, the $\lfloor k / 2\rfloor$-profiles of $z_{\ell}, z_{r}$ are determined by the $k$-profile of $z$ (see Figure 4). By Fact 4.6, this means that $z_{\ell}, z_{r}$ as well

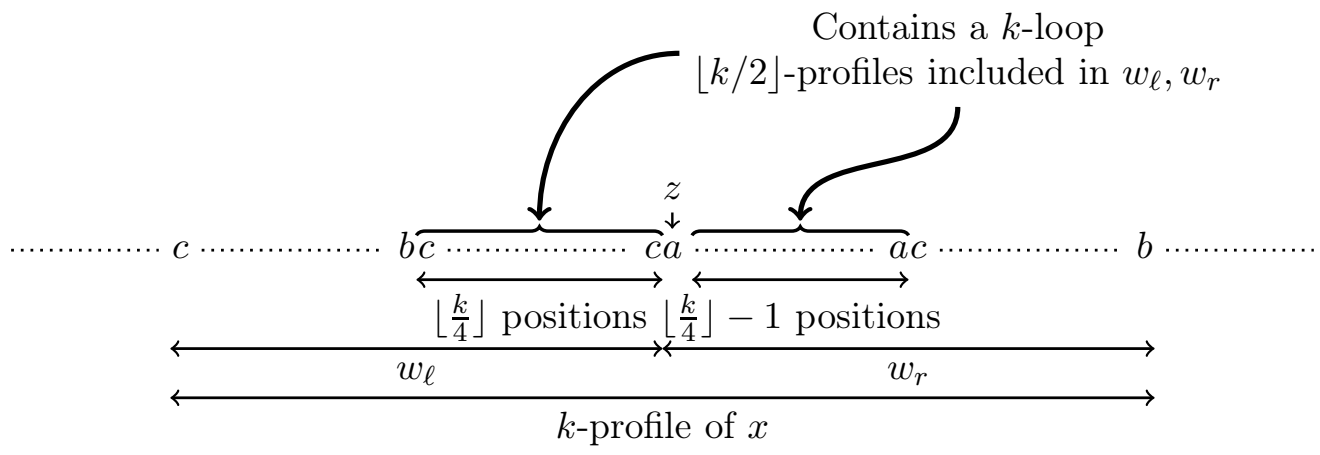

Figure 4: Construction in Lemma 4.9

as their actual $k$-loop are determined by the $k$-profile of $z$, which terminates the proof.

Taking $x, y$ as the first positions (resp. last positions) of $w_{1}, w_{2}$, Lemma 4.9 implies that $\mathfrak{p}=\left(u_{0}, v_{1}\right)=\left(u_{0}^{\prime}, v_{1}^{\prime}\right)\left(\right.$ resp. $\left.\mathfrak{s}=\left(v_{n}, u_{n}\right)=\left(v_{m}^{\prime}, u_{m}^{\prime}\right)\right)$. We finish by proving that for every block $\left(v_{\ell}, u, v_{r}\right)$ the number of indices $i$ such that $\left(v_{\ell}, u, v_{r}\right)=\left(v_{i}, u_{i}, v_{i+1}\right)$ is the same in the decompositions of $w_{1}^{\prime}, w_{2}^{\prime}$ up to threshold $d$. By definition of $(\mathfrak{p}, f, \mathfrak{s})$ this will prove that the decomposition of $w_{2}^{\prime}$ is indeed a $\mathcal{P}$-decomposition.

Let $\mathfrak{b}=\left(v_{\ell}, u, v_{r}\right)$ be a block. By Lemma 4.9, there exists a set $P$ of $k$-profiles such that the number of indices $i$ in $w_{1}^{\prime}$ (resp. $\left.w_{2}^{\prime}\right)$ such that $\mathfrak{b}=\left(v_{i}, u_{i}, v_{i+1}\right)$ is exactly the number of positions in $w_{1}$ (resp. in $w_{2}$ ) with a $k$-profile in $P$. Because $w_{1} \equiv_{k}^{d} w_{2}$ these numbers are then equal in $w_{1}, w_{2}$ up to threshold $d$ and this finishes the proof of Proposition 4.5.

\section{Separation by LTT Languages}

This section is devoted to LTT. Again, our theorem actually contains several results. In the case of LTT, two parameters are involved: the size $k$ of profiles and the counting threshold $d$. The first result in our theorem states that the bound on $k$ of Theorem 4.1 still holds for full LTT. This means that two languages are LTT-separable if and only if there exists some counting threshold $d$ such that they are LTT $[k, d]$-separable with the same bound $k$ as in Theorem 4.1. It turns out that this already yields an algorithm for testing LTT-separability. The algorithm relies on the decidability of Presburger arithmetic and is actually adapted in a straightforward manner from an algorithm of [4] for deciding membership in LTT.

While this first result gives an algorithm for testing separability, it gives no insight about an actual separator. Indeed, the procedure does not produce the actual counting threshold $d$. 
This is obtained in the second part of our theorem: we prove that two languages are LTTseparable if and only if they are LTT $[k, d]$-separable, where $k$ is as defined in Theorem 4.1. and $d$ is bounded by a function of the size of the monoid (or automaton) recognizing the input languages. Note that this result also gives another (brute-force) algorithm for testing LTT-separability. We now state our theorem. Recall that $A_{k}$ denotes the set of $k$-profiles.

Theorem 5.1. Let $L_{1}, L_{2}$ be regular languages. Let $\alpha: A^{*} \rightarrow M$ be a morphism into a finite monoid $M$ recognizing both $L_{1}$ and $L_{2}$. Let $\mathcal{A}$ be an $N F A$ recognizing both $L_{1}$ and $L_{2}$, such that $L_{i}=L\left(\mathcal{A}, I_{i}, F_{i}\right)$. Set $n$ to be either $|M|+1$ or $|\mathcal{A}|+1$. Let $k=4(|M|+1)$ and let $d=\left(\left|A_{k}\right| n\right)^{\left|A_{k}\right|}$. Then, the following conditions are equivalent:

(1) $L_{1}$ and $L_{2}$ are LTT-separable.

(2) There exists $d^{\prime} \in \mathbb{N}$ such that $L_{1}$ and $L_{2}$ are LTT $\left[k, d^{\prime}\right]$-separable.

(3) There exists $d^{\prime} \in \mathbb{N}$ such that no pair in $\alpha\left(L_{1}\right) \times \alpha\left(L_{2}\right)$ has a common $d^{\prime}$-pattern.

(4) There exists $d^{\prime} \in \mathbb{N}$ such that no pair in $\left(I_{1} \times F_{1}\right) \times\left(I_{2} \times F_{2}\right)$ has a common d'-pattern.

(5) $L_{1}$ and $L_{2}$ are LTT $[k, d]$-separable.

(6) The language $\left[L_{1}\right]_{k}^{d}$ separates $L_{1}$ from $L_{2}$.

Observe that decidability of LTT-separability is immediate from Item (5) by using the usual brute-force algorithm. As it was the case for a fixed counting threshold, this algorithm is slow and we will present a faster algorithm by using Items (3) and (4) in Section 6 ,

Corollary 5.2. It is decidable whether two given regular languages are LTT-separable.

By definition, a language is LTT if it is $\operatorname{LTT}[k, d]$ for some natural numbers $k, d$. Hence, the equivalence between Items (11), (2), (3) and (4) is an immediate consequence of Theorem 4.1. It is also clear that (5) $\Longrightarrow$ (6) $\Longrightarrow$ (11). Therefore, we only need to prove Items (5) or (6) from one of the other properties, i.e., the bound on the threshold $d$. Unfortunately, these are exactly the items we need for Corollary 5.2. However, we will prove that by reusing an algorithm of [4], Corollary 5.2 can also be derived directly from Item (2).

The remainder of this section is organized in three subsections. We first explain how Corollary 5.2 can be derived from Item (2) without actually having to compute a bound on the counting threshold. Next, we prove our bound on the counting threshold in Theorem 5.1. Finally, we discuss the optimality of this bound in the last subsection.

5.1. Decidability of LTT-separability as a consequence of Theorem 4.1. As we explained, the equivalence of Item (2) to LTT-separability is immediate from Theorem 4.1, We explain how to combine Item (2) with an algorithm of [4] to obtain decidability directly.

In [4], it is proved that once $k$ is fixed, Parikh's Theorem [27] can be used to prove that whether a language is $\operatorname{LTT}[k, d]$ for some $d$ can be rephrased as a computable Presburger formula. Decidability of membership in LTT can then be reduced to decidability of Presburger Arithmetic. For achieving this, two ingredients were needed: $a$ ) a bound on $k$, and b) the translation to Presburger arithmetic. It turns out that in [4], only the proof of $a$ ) was specific to membership. On the other hand, separation was already taken care of in $b$ ), because the intuition behind the Presburger formula was testing separability between the input language and its complement. In our setting, we have already replaced $a$ ), i.e., bounding $k$, by Item (2). Therefore, the argument can be generalized. We explain in the remainder of this subsection how to construct the Presburger formula. The argument makes use of the notion of commutative image, which we first recall. 
Commutative Images. Let $w \in A^{*}$. The commutative image of $w$, denoted by $\pi(w)$, is the $A$-indexed vector of natural numbers counting, for every $a \in A$, how many occurrences of $a$ there are in $w$. This notion can be easily generalized in order to count profiles rather than just letters. Let $k \in \mathbb{N}$. The $k$-image of $w, \pi_{k}(w)$, is the $A_{k}$-indexed vector of numbers counting for every $k$-profile $\left(w_{\ell}, w_{r}\right)$ the number of positions in $w$ with $k$-profile $\left(w_{\ell}, w_{r}\right)$. If $L$ is a language, the $k$-image of $L, \pi_{k}(L)$ is the set $\left\{\pi_{k}(w) \mid w \in L\right\}$. The definition of $\equiv_{k}^{d}$ yields the following fact.

Fact 5.3. Let $w, w^{\prime} \in A^{*}$ and let $k, d \in \mathbb{N}$. Then $w \equiv_{k}^{d} w^{\prime}$ if and only if $\pi_{k}(w)$ and $\pi_{k}\left(w^{\prime}\right)$ are equal componentwise up to threshold $d$.

A well-known result about commutative images is Parikh's Theorem [27], which states that if $L$ is context-free (and so in particular if $L$ is regular), then $\pi(L)$ is semilinear, i.e., Presburger definable [15]. As explained in [4], Parikh's Theorem extends without difficulty to $k$-images.

Theorem 5.4. Let $L$ be a context-free language and let $k \in \mathbb{N}$. Then $\pi_{k}(L)$ is semilinear. Moreover, a Presburger formula for this semilinear set can be computed from $L$.

Proof. When $k=1$, the proposition is Parikh's Theorem. When $k>1$, consider the language $L^{\prime}$ over the alphabet $A_{k}$ of $k$-profiles such that $w^{\prime} \in L^{\prime}$ if and only if there exists $w \in L$ of the same length and such that a position in $w^{\prime}$ is labeled by the $k$-profile of the same position in $w$. It is straightforward to see that $L^{\prime}$ is context-free and that the $k$-image $\pi_{k}(L)$ of $L$ is its commutative image $\pi\left(L^{\prime}\right)$, which is semilinear by Parikh's Theorem.

We can now explain how to decide LTT-separability. By Item (2) in Theorem 5.1, $L_{1}, L_{2}$ are LTT-separable if and only if they are $\operatorname{LTT}[k, d]$-separable for $k=4(|M|+1)$ (where $M$ is a monoid recognizing both $\left.L_{1}, L_{2}\right)$ and some natural number $d$. Therefore, whether $L_{1}, L_{2}$ are LTT-separable can be rephrased as follows: does there exist some threshold $d$ such that there exist no words $w_{1} \in L_{1}, w_{2} \in L_{2}$ such that $w_{1} \equiv_{k}^{d} w_{2}$ ? By Fact 5.3, this can be expressed in terms of $k$-images: does there exist a threshold $d$ such that there exist no vectors of natural numbers $\bar{x}_{1} \in \pi_{k}\left(L_{1}\right), \bar{x}_{2} \in \pi_{k}\left(L_{2}\right)$ that are equal up to threshold $d$ ? It follows from Theorem 5.4 that the above question can be expressed as a computable Presburger formula. Decidability of LTT-separability then follows from decidability of Presburger Arithmetic.

5.2. Bounding the Counting Threshold. We now prove the bound on the counting threshold $d$. This amounts to proving that Items (5) and (6) in Theorem 5.1 are equivalent to Item (2). By definition, it is immediate that $(\sqrt{6}) \Rightarrow(5) \Rightarrow(2)$. It remains to prove (2) $\Rightarrow$ (6) .

We actually prove the contrapositive: if $\left[L_{1}\right]_{k}^{d}$ does not separate $L_{1}$ from $L_{2}$ for the values of $k, d$ defined in the theorem, then there is no $\operatorname{LTT}\left[k, d^{\prime}\right]$-separator for any $d^{\prime}$.

Assume that $\left[L_{1}\right]_{k}^{d}$ is not a separator. By definition, this means that there exist $w_{1} \in L_{1}$ and $w_{2} \in L_{2}$ such that $w_{1} \equiv_{k}^{d} w_{2}$. Set $d^{\prime}$ some arbitrary natural number. We prove that $d$ is large enough to construct words $w_{1}^{\prime} \in L_{1}, w_{2}^{\prime} \in L_{2}$ such that $w_{1}^{\prime} \equiv_{k}^{d^{\prime}} w_{2}^{\prime}$. By definition of $\equiv_{k}^{d^{\prime}}$, this means that there exists no LTT $\left[k, d^{\prime}\right]$-separator, which is what we want to prove.

Recall that $n=|M|+1$ or $n=|\mathcal{A}|+1$. To simplify the writing, we only treat the case when $n=|\mathcal{A}|+1$. The other case can be proved similarly. Set $m=\left|A_{k}\right| n$, so that $d=m^{\left|A_{k}\right|}$. 
For $\ell \leqslant\left|A_{k}\right|$, we prove the following property by induction:

If $u_{1} \in L_{1}, u_{2} \in L_{2}$ are such that $u_{1} \equiv_{k}^{m^{\ell}} u_{2}$, then for all $d^{\prime}$, if the number of $\mathcal{P}(\ell) \quad k$-profiles that do not occur more than $d^{\prime}$ times in both $u_{1}$ and $u_{2}$ is smaller than $\ell$, then there exist words $u_{1}^{\prime} \in L_{1}$ and $u_{2}^{\prime} \in L_{2}$ such that $u_{1}^{\prime} \equiv_{k}^{d^{\prime}} u_{2}^{\prime}$.

Before proving $\mathcal{P}(\ell)$, note that by definition of $d=m^{\left|A_{k}\right|}$ and since $A_{k}$ is the set of all $k$-profiles, $w_{1}, w_{2}$ verify the premise of $\mathcal{P}\left(\left|A_{k}\right|\right)$. Therefore, $\mathcal{P}\left(\left|A_{k}\right|\right)$ entails that there exist words $w_{1}^{\prime}, w_{2}^{\prime}$ such that the desired property $w_{1}^{\prime} \equiv_{k}^{d^{\prime}} w_{2}^{\prime}$ holds for all $d^{\prime}$. It remains to prove $\mathcal{P}(\ell)$ for $\ell \leqslant\left|A_{k}\right|$, which we do by induction on $\ell$.

The main idea for the inductive step is that by choice of $d, k$-profiles that occur many times in $w_{1}, w_{2}$ can be pumped in more than $d^{\prime}$ occurrences. This is summarized in the following lemma, that we prove below.

Lemma 5.5. Let $d^{\prime} \in \mathbb{N}$. Let $h \geqslant 1$ be a natural number, $h^{\prime} \geqslant n\left|A_{k}\right| h$ and $w \in L_{1}$ (resp. $\left.w \in L_{2}\right)$. One can construct a word $w^{\prime} \in L_{1}$ (resp. $\left.w^{\prime} \in L_{2}\right)$ such that $w^{\prime} \equiv_{k}^{h} w$ and every $k$-profile that occurs $h^{\prime}$ or more times in $w$ occurs $d^{\prime}$ or more times in $w^{\prime}$.

Assume for a moment that Lemma 5.5 holds and let us deduce that $\mathcal{P}(\ell)$ holds, by induction on $\ell$. If $\ell=0$, the result is obvious since by definition all $k$-profiles in $u_{1}, u_{2}$ occur more than $d^{\prime}$ times in both words and therefore $u_{1} \equiv_{k}^{d^{\prime}} u_{2}$.

Assume now that $\ell>0$. If $u_{1} \equiv_{k}^{d^{\prime}} u_{2}$, then it suffices to take $u_{1}^{\prime}=u_{1}$ and $u_{2}^{\prime}=u_{2}$. Otherwise, since $u_{1} \equiv_{k}^{m^{\ell}} u_{2}$, this means that there exists at least one $k$-profile $\left(w_{\ell}, w_{r}\right)$ that occurs more than $m^{\ell}$ times in both $u_{1}$ and $u_{2}$ but less than $d^{\prime}$ times in at least one of the two words. By applying Lemma 5.5 to both $u_{1}$ and $u_{2}$ with $h=m^{\ell-1}$ and $h^{\prime}=m^{\ell}$, we get two words $u_{1}^{\prime \prime} \in L_{1}$ and $u_{2}^{\prime \prime} \in L_{2}$ such that $u_{1}^{\prime \prime} \equiv_{k}^{h} u_{2}^{\prime \prime}$. Moreover, $\left(w_{\ell}, w_{r}\right)$ now occurs more than $d^{\prime}$ times in both $u_{1}^{\prime \prime}$ and $u_{2}^{\prime \prime}$. Therefore, the number of $k$-profiles that do not occur more than $d^{\prime}$ times in both $u_{1}^{\prime \prime}$ and $u_{2}^{\prime \prime}$ is smaller than $\ell-1$. Hence, we can apply the induction hypothesis to $u_{1}^{\prime \prime}$ and $u_{2}^{\prime \prime}$ which yields the desired $u_{1}^{\prime}, u_{2}^{\prime}$.

To conclude the proof, it remains to show Lemma 5.5.

Proof of Lemma 5.5. By symmetry, we may assume that $w \in L_{1}$. The intuition is that as soon as a $k$-profile occurs more than $|\mathcal{A}|+1$ times (which is the case if it occurs more than $n$ times), there exist two occurrences of this $k$-profile that are labeled with the same state in the run of $\mathcal{A}$ on $w$. Therefore, pumping can be used on $w$ to generate $d^{\prime}$ copies of the $k$-profile without affecting membership in $L_{1}$. The issue with this argument is that in order to enforce $w^{\prime} \equiv_{k}^{h} w$, we need to be careful and avoid duplicating $k$-profiles that occur less than $h$ times in $w$. This is why we actually need a much higher constant than $n$ in order to achieve the pumping.

Let $\left(w_{\ell}, w_{r}\right)$ be some $k$-profile that occurs more than $h^{\prime}$ times in $w$ (if there is none, it suffices to take $w^{\prime}=w$ ). We explain how $w$ can be pumped in $w^{\prime}$ that contains more than $d^{\prime}$ copies of $\left(w_{\ell}, w_{r}\right)$ while enforcing $w^{\prime} \equiv_{k}^{h} w$. The construction can then be repeated to treat all $k$-profiles occurring more than $h^{\prime}$ times in $w$, in order to get the desired $w^{\prime}$.

Let $x_{1}<\cdots<x_{h^{\prime}}$ be $h^{\prime}$ positions where $\left(w_{\ell}, w_{r}\right)$ occurs. Observe that there are at most $\left|A_{k}\right|(h-1)$ positions in $w$ such that the $k$-profile at this position occurs strictly less than $h$ times in $w$. By choice of $h^{\prime} \geqslant n\left|A_{k}\right| h$, a simple application of the pigeonhole principle yields that there exist at least $n$ consecutive positions in the list, say $x_{i}, \ldots, x_{i+(n-1)}$, such that no intermediate position between $x_{i}$ and $x_{i+(n-1)}$ has a $k$-profile occurring less than $h$ times in $w$. By choice of $n$, there are two positions among $x_{i}, \ldots, x_{i+(n-1)}$ that are labeled with 
the same state in the run of $\mathcal{A}$ on $w$. Therefore, the corresponding infix can be pumped to generate $d^{\prime}$ copies of $\left(w_{\ell}, w_{r}\right)$ without affecting membership in $L_{1}$. Moreover, by choice of the positions $x_{i}, \ldots, x_{i+(n-1)}$, the pumping did not duplicate $k$-profiles occurring less than $h$ times in $w$. Therefore, the resulting word $w^{\prime}$ verifies $w^{\prime} \equiv_{k}^{h} w$ and $w^{\prime} \in L_{1}$.

5.3. Optimality of the Counting Threshold. Observe that the bound of Theorem 5.1 for the counting threshold is exponential in the size of $\left|A_{k}\right|$ (which is itself exponential in the size of the monoid). A relevant question is to know whether this bound can be improved.

Our proof completely separates the bounding of $k$ and $d$. We first provide a bound on $k$ in Theorem 4.1. Then, we bound the threshold by essentially viewing our words as words over the alphabet $A_{k}$ of $k$-profiles. This technique ignores properties of $k$-profiles. In particular, the $k$-profiles of adjacent positions are strongly related, a fact that our proof does not exploit.

In this subsection, we prove that getting a better bound on the counting threshold would require taking these additional properties into account. More precisely, we prove that if $k=1$ (which means that the $k$-profile of a position is just its label), we can construct separable languages for which the separator requires a counting threshold that is exponential in $|A|$.

For convenience, we assume the alphabet $A$ to be of even size and write $A=\left\{a_{1}, \ldots, a_{2 m}\right\}$. Consider the following languages

$$
\begin{aligned}
& L_{1}=a_{1} \cdot\left(a_{2} a_{3} a_{3}\right)^{*}\left(a_{4} a_{5} a_{5}\right)^{*} \cdots\left(a_{2 m-2} a_{2 m-1} a_{2 m-1}\right)^{*} \cdot\left(a_{2 m} a_{2 m} a_{2 m}\right)^{*}, \\
& L_{2}=\left(a_{1} a_{2} a_{2}\right)^{*}\left(a_{3} a_{4} a_{4}\right)^{*} \cdots\left(a_{2 m-1} a_{2 m} a_{2 m}\right)^{*} .
\end{aligned}
$$

Lemma 5.6. $L_{1}, L_{2}$ are LTT $[1, d]$-separable for some $d$, but not LTT $\left[1,2^{2 m-1}\right]$-separable.

Proof. We prove that $L_{1}, L_{2}$ are LTT $[1, d]$-separable for $d=2^{2 m-1}+1$. Consider the following language $L$. A word $w$ belongs to $L$ if and only if for all odd $i$ either $w$ contains at least $2^{i-1}+1$ copies of $a_{i}$, or the number of copies of $a_{i+1}$ in $w$ is exactly twice the number of copies of $a_{i}$ in $w$.

The maximal threshold up to which we have to count occurrences of letters for checking membership in $L$ of a word $w$ is the case where $w$ has exactly $2^{2 m-2}$ occurrences of $a_{2 m-1}$, and therefore exactly $2^{2 m-1}$ occurrences of $a_{2 m}$. This shows that $L \in \operatorname{LTT}\left[1,2^{2 m-1}+1\right]$.

We prove that $L$ is a separator. By definition, we have $L \supseteq L_{2}$. We prove that $L \cap L_{1}=\varnothing$. By contradiction, assume that $w \in L \cap L_{1}$. Since $w \in L_{1}$, it contains only one copy of $a_{1}$. Then, since $w \in L$, it must contain two copies of $a_{2}$. By iterating the argument we get that $w$ must contain $2^{2 m-1}$ copies of $a_{2 m}$, which is impossible since this number must be multiple of 3 , by definition of $L_{1}$. It follows that $L \in \operatorname{LTT}\left[1,2^{2 m-1}+1\right]$ is a separator.

It remains to prove that $L_{1}, L_{2}$ are not $\operatorname{LTT}\left[1,2^{2 m-1}\right]$-separable. Consider the words

$$
\begin{array}{ll}
w_{1}=a_{1}\left(a_{2} a_{3}^{2}\right)^{2} \cdots\left(a_{2 m-2} a_{2 m-1}^{2}\right)^{2^{2 m-3}}\left(a_{2 m}\right)^{3 \times 2^{2 m-1}} & \in L_{1}, \\
w_{2}=\left(a_{1} a_{2}^{2}\right)\left(a_{3} a_{4}^{2}\right)^{4} \cdots\left(a_{2 m-1} a_{2 m}^{2}\right)^{2^{2 m-2}} & \in L_{2} .
\end{array}
$$

It is clear that $w_{1} \equiv_{1}^{2 m-1} w_{2}$. Therefore $L_{1}, L_{2}$ are not LTT $\left[1,2^{2 m-1}\right]$-separable. 


\section{COMPleXity: UPPER AND LOWER BOUNDS}

In this section, we present lower and upper complexity bounds for deciding LT- and LTTseparability.

Both the lower and upper bounds rely on the pattern criteria of Theorems 4.2 and 5.1 We are able to prove that starting from NFAs or DFAs recognizing the input languages, deciding separability can be achieved in CO-NEXPTIME for LT and in 2-ExPSPACE for LTT. Moreover, we prove a CO-NP lower bound for both problems.

6.1. Upper Bounds. In this subsection, we prove the complexity upper bounds for the separation problem for both LT and LTT. The corresponding algorithms rely on the patterns criteria of Theorems 4.2 and 5.1. We prove the two following results.

Proposition 6.1. Deciding whether two languages accepted by some NFA are LT-separable can be achieved in CO-NEXPTIME.

Proposition 6.2. Deciding whether two languages accepted by some NFA are LTT-separable can be achieved in 2-EXPSPACE.

Both algorithms work by reducing the problems to the special case of $k=1$, i.e., whether there exists an LT (resp. LTT) separator that considers only 1-profiles. The reduction is identical in both cases and the difference resides in proving that it is correct. These proofs rely on Item (5) in Theorem 4.2 and Item (4) in Theorem 5.1 respectively. The computations involved in the reduction can be done in ExPTIME and the new NFA it outputs is of size exponential in the input NFA. Therefore, it then suffices to give algorithms for the special case $k=1$, which run in CO-NP for LT and ExPSPACE for LTT.

Note that a reduction to $k=1$ could also be done by considering the bound $k$ on the size of profiles in Theorems 4.2 and 5.1 . Indeed, once $k$ is fixed it suffices to modify the input NFA to work on the alphabet of $k$-profiles to be reduced to the case $k=1$. However, this technique might yield an NFA that is doubly exponential in the size of the input NFA.

We first present and prove the reduction to the case $k=1$. Then we explain how to decide both problems in this special case.

6.1.1. Reduction to the case $k=1$. Let $\mathcal{A}=(Q, A, \delta)$ be an NFA. It follows from Theorem 4.2 (resp. Theorem 5.1) that to determine whether $L\left(\mathcal{A}, I_{1}, F_{1}\right)$ and $L\left(\mathcal{A}, I_{2}, F_{2}\right)$ are not LT-separable (resp. not LTT-separable), it suffices to verify whether there exists a pair in $\left(I_{1} \times F_{1}\right) \times\left(I_{2} \times F_{2}\right)$ having a common 1-pattern (resp. whether there exists $d$ such that some pair in $\left(I_{1} \times F_{1}\right) \times\left(I_{2} \times F_{2}\right)$ has a common $d$-pattern $)$. This requires verifying whether there exist a pattern $\mathcal{P}$ and $\mathcal{P}$-decompositions compatible with such a pair.

Observe that a $\mathcal{P}$-decomposition can be viewed as a word over the alphabet of blocks. The main idea behind the reduction is to construct a new NFA $\widetilde{\mathcal{A}}$ recognizing words over the alphabet of blocks that represent $\mathcal{P}$-decompositions that, for some $\mathcal{P}$, are compatible with pairs of states of $I_{1} \times F_{1}$ and of $I_{2} \times F_{2}$ in $\mathcal{A}$. There are two main issues with this outline. First, the alphabet of blocks is infinite. Second, a notion of compatibility has to be enforced between consecutive blocks in a word, i.e., $\left(v, u, v^{\prime}\right)$ needs to be followed by $\left(v^{\prime}, u^{\prime}, v^{\prime \prime}\right)$ for some $u^{\prime}, v^{\prime \prime}$. Again, this compatibility cannot be simply encoded in the states since there are infinitely many words.

Both issues are solved using a similar argument. Recall that we are only interested in $\mathcal{P}$-decompositions that are compatible with pairs of states of $I_{1} \times F_{1}$ and of $I_{2} \times F_{2}$ in $\mathcal{A}$. 
Observe that for a block $\left(v, u, v^{\prime}\right)$ to appear in such a decomposition, there need to exist states $q, q^{\prime}$ with loops at $q, q^{\prime}$ labeled by $v, v^{\prime}$ respectively and a path from $q$ to $q^{\prime}$ labeled by $u$. We abstract a block by the set of pairs of states verifying this property. Since there are finitely many such sets, this yields a finite alphabet. The same argument can be used for compatibility: all words $v$ that need to be considered for compatibility need to label a loop at some state $q$. We abstract $v$ by the set of states having such a loop labeled $v$. The information can then be encoded in the states of $\widetilde{\mathcal{A}}$.

We now describe the formal construction. Let $R \subseteq Q$. We say that $R$ is synchronizable if there exists a nonempty word $v \in A^{*}$ such that for all $q \in R$ there exists a loop at $q$ labeled by $v$. We will encode compatibility in synchronizable sets of states.

If $T \subseteq Q^{2}$, we denote by $\ell(T)$ (resp. by $r(T)$ ) the set of states that are left (resp. right) members of pairs in $T$. We say that $T$ is synchronizable if a) there exists $u \in A^{*}$ such that for all $\left(q, q^{\prime}\right) \in T$ there exists a run from $q$ to $q^{\prime}$ labeled by $\left.u, \mathbf{b}\right) \ell(T)$ is synchronizable and $\mathbf{c}$ ) $r(T)$ is synchronizable. In order to deal with prefixes and suffixes, we generalize the notion to these limit cases. We say that $T$ is prefix synchronizable (resp. suffix synchronizable) if a) and c) (resp. a) and b)) hold. Finally, we say that $T$ is weakly synchronizable if a) holds. We set $B_{w}, B_{p}, B_{i}$ and $B_{s}$ as the sets of weakly synchronizable, prefix synchronizable, synchronizable and suffix synchronizable sets of pairs of states, respectively.

We can now define $\widetilde{\mathcal{A}}$. Set the alphabet $B$ as the disjoint union of $B_{w}, B_{p}, B_{i}$ and $B_{s}$. The state set $\widetilde{Q}$ is defined as follows.

$$
\widetilde{Q}=\{(r, R) \mid r \in R \subseteq Q, R \text { is synchronizable, } r \in Q\} \cup I_{1} \cup F_{1} \cup I_{2} \cup F_{2} .
$$

We now define the transitions. For all $b \in B_{w}$, we add a $b$-transition from $q_{k} \in I_{k}$ to $r_{k} \in F_{k}$ if $\left(q_{k}, r_{k}\right) \in b$. For all $b \in B_{p}$, we add a $b$-transition from $q_{k} \in I_{k}$ to $\left(r_{k}, R_{k}\right)$ if $\left(q_{k}, r_{k}\right) \in b$ and $R_{k}=r(b)$. Similarly, for all $b \in B_{s}$, we add a $b$-transition from $\left(r_{k}, R_{k}\right)$ to $q_{k} \in F_{k}$ if $\left(r_{k}, q_{k}\right) \in b$ and $R_{k}=\ell(b)$. Finally, for all $b \in B_{i}$, we add a $b$-transition from $\left(r_{k}, R_{k}\right)$ to $\left(s_{k}, S_{k}\right)$ if $\left(r_{k}, s_{k}\right) \in b, R_{k}=\ell(b)$ and $S_{k}=r(b)$.

This ends the construction of $\widetilde{\mathcal{A}}$ from $\mathcal{A}, I_{1}, F_{1}, I_{2}, F_{2}$. Observe that it is of size exponential in the size of $\mathcal{A}$. We now prove that the computation can be done in ExpTIME.

Lemma 6.3. Given an NFA $\mathcal{A}, I_{1}, F_{1}, I_{2}, F_{2}$ as input, $\widetilde{\mathcal{A}}$ can be constructed in ExpTIME.

Proof. Testing synchronizability of a set of states (resp. a set of pairs of states) can easily be reduced to checking nonempty intersection between a set of NFAs. This is known to be a PSPACE-complete problem. It follows that computing the synchronizable sets can be done in Exptime. It is then clear that the remaining computations can be done in Exptime.

We now prove that the construction is correct, i.e., that it reduces LT- and LTTseparability to the special case $k=1$.

Lemma 6.4. Let $\mathcal{A}=(Q, A, \delta)$ be an $N F A$, and let $I_{1}, F_{1}, I_{2}, F_{2} \subseteq Q$. Then:

(1) $L\left(\mathcal{A}, I_{1}, F_{1}\right), L\left(\mathcal{A}, I_{2}, F_{2}\right)$ are LT-separable if and only if $L\left(\widetilde{\mathcal{A}}, I_{1}, F_{1}\right), L\left(\widetilde{\mathcal{A}}, I_{2}, F_{2}\right)$ are $\mathrm{LT}[1]$-separable.

(2) $L\left(\mathcal{A}, I_{1}, F_{1}\right), L\left(\mathcal{A}, I_{2}, F_{2}\right)$ are LTT-separable if and only if there exists $d \in \mathbb{N}$ such that $L\left(\widetilde{\mathcal{A}}, I_{1}, F_{1}\right), L\left(\widetilde{\mathcal{A}}, I_{2}, F_{2}\right)$ are LTT $[1, d]$-separable.

Proof. We only give the proof for LTT using Condition (4) from Theorem 5.1. The proof for LT is obtained similarly using Condition (5) from Theorem 4.2 . 
Suppose that $L\left(\mathcal{A}, I_{1}, F_{1}\right)$ and $L\left(\mathcal{A}, I_{2}, F_{2}\right)$ are not LTT-separable. We prove that for all $d \in \mathbb{N}, L\left(\widetilde{\mathcal{A}}, I_{1}, F_{1}\right)$ and $L\left(\widetilde{\mathcal{A}}, I_{2}, F_{2}\right)$ are not LTT $[1, d]$-separable. Set $d \in \mathbb{N}$. By Condition (4) of Theorem 5.1, there exists a common $d$-pattern $\mathcal{P}$ : there are two words $w_{1} \in L\left(\mathcal{A}, I_{1}, F_{1}\right)$ and $w_{2} \in L\left(\mathcal{A}, I_{2}, F_{2}\right)$ such that $w_{1}=u_{0} v_{1} u_{1} \ldots v_{n} u_{n}$ and $w_{2}=u_{0}^{\prime} v_{1}^{\prime} u_{1}^{\prime} \ldots v_{m}^{\prime} u_{m}^{\prime}$ are $\mathcal{P}_{-}$ decompositions, respectively compatible with some $\left(q_{1}, r_{1}\right) \in I_{1} \times F_{1}$ in $\mathcal{A}$ and $\left(q_{2}, r_{2}\right) \in$ $I_{2} \times F_{2}$ in $\mathcal{A}$. There are two cases.

In the first case, $n=m=0$ and $w_{1}=w_{2}$. Therefore, $L\left(\mathcal{A}, I_{1}, F_{1}\right) \cap L\left(\mathcal{A}, I_{2}, F_{2}\right) \neq \varnothing$. By definition, it follows that there exists some $b \in B_{w}$ such that $b \in L\left(\widetilde{\mathcal{A}}, I_{1}, F_{1}\right) \cap L\left(\widetilde{\mathcal{A}}, I_{2}, F_{2}\right)$, which ends the proof.

In the second case, $n, m>0$. Set $\widehat{w}_{1}=\mathfrak{p} \mathfrak{b}_{1} \cdots \mathfrak{b}_{n-1} \mathfrak{s}$ such that $\mathfrak{p}=\left(u_{0}, v_{1}\right), \mathfrak{s}=\left(u_{n}, v_{n}\right)$ and for all $i, \mathfrak{b}_{i}=\left(v_{i}, u_{i}, v_{i+1}\right)$. Similarly, we define $\widehat{w}_{2}=\mathfrak{p}^{\prime} \mathfrak{b}_{1}^{\prime} \cdots \mathfrak{b}_{m-1}^{\prime} \mathfrak{s}^{\prime}$. Observe that since we started from $\mathcal{P}$-decompositions for a $d$-pattern $\mathcal{P}$, we have $\widehat{w}_{1} \equiv_{1}^{d} \widehat{w}_{2}$. We use these words to construct $\widetilde{w}_{1}, \widetilde{w}_{2} \in B^{*}$ belonging to $L\left(\widetilde{\mathcal{A}}, I_{1}, F_{1}\right)$ and $L\left(\widetilde{\mathcal{A}}, I_{2}, F_{2}\right)$, respectively.

Let $\mathfrak{b}$ be a block appearing in $\widehat{w}_{1}, \widehat{w}_{2}$. We set $T_{\mathfrak{b}} \subseteq Q^{2}$ as the set of pairs of states that correspond to $\mathfrak{b}$ in the runs on $w_{1}$ and $w_{2}$. Since the $\mathcal{P}$-decompositions are compatible with their respective NFAs, by definition, $T_{\mathfrak{b}}$ is synchronizable. Similarly, if $\mathfrak{p}$ (resp. $\mathfrak{s}$ ) is a prefix (resp. suffix) block occurring in $\widehat{w}_{1}, \widehat{w}_{2}$, we get a prefix (resp. suffix) synchronizable set $T_{\mathfrak{p}}$ (resp. $T_{\mathfrak{s}}$ ). We now set $\widetilde{w}_{1}=T_{\mathfrak{p}} T_{\mathfrak{b}_{1}} \cdots T_{\mathfrak{b}_{n-1}} T_{\mathfrak{s}}$ and $\widetilde{w}_{2}=T_{\mathfrak{p}^{\prime}} T_{\mathfrak{b}_{1}^{\prime}} \cdots T_{\mathfrak{b}_{m-1}^{\prime}} T_{\mathfrak{s}^{\prime}}$. By definition of $\widetilde{\mathcal{A}}$ and the fact that the $\mathcal{P}$-decompositions are $\left(q_{1}, r_{1}\right)$-, $\left(q_{2}, r_{2}\right)$-compatible, $\widetilde{w}_{1}, \widetilde{w}_{2} \in B^{*}$ belong to $L\left(\widetilde{\mathcal{A}}, I_{1}, F_{1}\right)$ and $L\left(\widetilde{\mathcal{A}}, I_{2}, F_{2}\right)$, respectively. Moreover, since $\widehat{w}_{1} \equiv_{1}^{d} \hat{w}_{2}$, we have $\widetilde{w}_{1} \equiv_{1}^{d} \widetilde{w}_{2}$. We conclude that $L\left(\widetilde{\mathcal{A}}, I_{1}, F_{1}\right)$ and $L\left(\widetilde{\mathcal{A}}, I_{2}, F_{2}\right)$ are not LTT $[1, d]$-separable.

Conversely, assume that for all $d \in \mathbb{N}, L\left(\widetilde{\mathcal{A}}, I_{1}, F_{1}\right)$ and $L\left(\widetilde{\mathcal{A}}, I_{2}, F_{2}\right)$ are not LTT $[1, d]$ separable. We prove that for all $d \in \mathbb{N}$, there exists a pair in $\left(I_{1} \times F_{1}\right) \times\left(I_{2} \times F_{2}\right)$ having a common $d$-pattern $\mathcal{P}$. Set $d \in \mathbb{N}$, by hypothesis, there exist $\widetilde{w}_{1}, \widetilde{w}_{2} \in B^{*}$ in $L\left(\widetilde{\mathcal{A}}, I_{1}, F_{1}\right), L\left(\widetilde{\mathcal{A}}, I_{2}, F_{2}\right)$, respectively, such that $\widetilde{w}_{1} \equiv_{1}^{d} \widetilde{w}_{2}$. Again, we have two cases. By definition of $\widetilde{\mathcal{A}}$, either $\widetilde{w}_{1}, \widetilde{w}_{2} \in B_{w}$ or $\widetilde{w}_{1}, \widetilde{w}_{2} \in B_{p}\left(B_{i}\right)^{*} B_{s}$. In the first case, this means that $L\left(\mathcal{A}, I_{1}, F_{1}\right) \cap L\left(\mathcal{A}, I_{2}, F_{2}\right) \neq \varnothing$, and therefore it suffices to set $\mathcal{P}$ as a word in this intersection to conclude.

Otherwise, $\widetilde{w}_{1}=b_{p} b_{1} \cdots b_{n} b_{s}$ and $\widetilde{w}_{2}=b_{p} b_{1}^{\prime} \cdots b_{m}^{\prime} b_{s}$. By definition of $B_{p}, B_{i}, B_{s}$, to each label appearing in $\widetilde{w}_{1}, \widetilde{w}_{2}$ we can associate a unique block, prefix block or suffix block. We set $\mathcal{P}=(\mathfrak{p}, f, \mathfrak{s})$ as the $d$-pattern defined in the following way: $\mathfrak{p}, \mathfrak{s}$ are the prefix and suffix blocks associated to $b_{p}$ and $b_{s}$ respectively. Since $\widetilde{w}_{1} \equiv_{1}^{d} \widetilde{w}_{2}$, for all blocks $\mathfrak{b}$, the number of occurrences of labels $b$ such that $\mathfrak{b}$ is associated to $b$ is the same in $\widetilde{w}_{1}, \widetilde{w}_{2}$ up to threshold $d$, we set $f(\mathfrak{b})$ as this number. Finally, let $p_{i} \in I_{i}$ and $q_{i} \in F_{i}$ such that $\widetilde{w}_{i}$ labels a path from $p_{i}$ to $q_{i}$ in $\widetilde{\mathcal{A}}$. It is now straightforward to verify that the pair $\left(\left(p_{1}, q_{1}\right),\left(p_{2}, q_{2}\right)\right)$ admits compatible $\mathcal{P}$-decompositions in $\mathcal{A}$, which terminates the proof.

6.1.2. Deciding the case $k=1$. We explain how LT- and LTT-separability can be decided when the size of profiles if fixed to 1. Observe that in this case, the 1-profile of a position is its label. We prove the two following lemmas.

Lemma 6.5. Deciding whether two regular languages given by an accepting NFA are LT[1]separable is in $\mathrm{CO}-\mathrm{NP}$.

Lemma 6.6. Given an NFA accepting two languages $L_{1}$ and $L_{2}$, deciding whether there exists $d \in \mathbb{N}$ such that $L_{1}$ and $L_{2}$ are LTT $[1, d]$-separable is in EXPSPACE. 
As we explained in Section 5, decidability follows from Parikh's Theorem and decidability of Presburger arithmetic. However, applying these results naively yields high complexity. We explain here how to refine the argument in order to get $\mathrm{CO}-\mathrm{NP}$ and ExPSPACE complexities.

Set $\pi\left(L_{1}\right), \pi\left(L_{2}\right)$ as the Parikh's images of $L_{1}$ and $L_{2}$, respectively. As we explained in Section 5, non-LTT-separability is equivalent to the following Presburger property: "for all $d \in \mathbb{N}$ there exist $\bar{x}_{1} \in \pi\left(L_{1}\right), \bar{x}_{2} \in \pi\left(L_{2}\right)$ that are equal componentwise up to threshold $d$." By [37, existential Presburger formulas for $\pi\left(L_{1}\right), \pi\left(L_{2}\right)$ can be computed in linear time (see also [47] for the same technique applied to context-free grammars). Therefore, a Presburger formula for the property can also be computed in linear time. Moreover, by definition, this property has exactly one quantifier alternation, it then follows from [35] that it can be decided in EXPSPACE.

The same construction can be done for LT. However, the counting threshold $d$ is fixed to 1. Therefore, the constructed formula is existential. It is known that existential Presburger formuals can be decided in NP (see [5, 48]). We conclude that LT[1]-separability is in $\mathrm{CO}-\mathrm{NP}$.

In the case of LT the problem is actually CO-NP-complete. This means that Lemma 6.5 cannot be improved and that improving Proposition 6.1 would require improving the reduction. For LTT, the situation is different, it is likely that a sharper analysis of the Presburger formula would yield a better upper bound. Indeed, while deciding Presburger formulas with only one quantifier alternation is already very costly in general, the formula we consider is very specific.

6.2. Lower Bounds. In this subsection, we prove CO-NP lower bounds for both LT and LTT-separability. The bounds hold when the input languages are given as NFAs or DFAs.

Proposition 6.7. Let $L_{1}, L_{2}$ be languages accepted by two input DFAs $\mathcal{A}_{1}, \mathcal{A}_{2}$, respectively. The two following problems are $\mathrm{CO}-\mathrm{NP}$-hard:

(1) Are $L_{1}$ and $L_{2}$ LT-separable?

(2) Are $L_{1}$ and $L_{2}$ LTT-separable?

Proof. We only do the proof for LTT. The reduction is identical for the LT case. We prove that testing whether $L_{1}$ and $L_{2}$ are not LTT-separable is NP-hard. The proof is by reduction of 3-SAT. From an instance of 3-SAT, we construct two DFAs and prove that the corresponding languages are not LTT-separable if and only if the 3-SAT instance is satisfiable.

Let $\mathcal{C}=\left\{C_{1}, \ldots, C_{m}\right\}$ be a set of 3 -clauses over the set of variables $\left\{x_{1}, \ldots, x_{n}\right\}$. We construct DFAs $\mathcal{A}_{1}$ and $\mathcal{A}_{2}$ over the alphabet $A:=\left\{\#, x_{1}, \ldots, x_{n}, \neg x_{1}, \ldots, \neg x_{n}\right\}$. Given $w \in A^{*}$, we say that $w$ encodes an assignment of truth values if for all $i \leqslant n, w$ contains either the label $x_{i}$ or the label $\neg x_{i}$, but not both. It is straightforward to see that an assignment of truth values for the variables can be uniquely defined from such a word. Moreover, by definition of LTT, we have the following fact.

Fact 6.8. The language of correct assignments is LTT.

Intuitively, we want our DFAs $\mathcal{A}_{1}, \mathcal{A}_{2}$ to verify the following three properties:

(1) all words accepted by $\mathcal{A}_{1}$ encode assignments, and for each assignment of variables, a word coding that assignment is accepted by $\mathcal{A}_{1}$. 
(2) all assignments accepted by $\mathcal{A}_{2}$ satisfy $\mathcal{C}$, and for each assignment of variables satisfying $\mathcal{C}$, a word coding that assignment is accepted by $\mathcal{A}_{2}$.

(3) if there exist $w_{1}, w_{2} \in A^{*}$ accepted by $\mathcal{A}_{1}, \mathcal{A}_{2}$ that encode the same assignment of variables, then for all $k, d \in \mathbb{N}, w_{1}, w_{2}$ can be chosen such that $w_{1} \equiv_{k}^{d} w_{2}$.

Conditions (1) and (2) are simple to enforce. Indeed, for Condition (11), it suffices to construct a DFA recognizing the words $t_{1} \ldots t_{n}$ such that for all $i \leqslant n, t_{i}=x_{i}$ or $t_{i}=\neg x_{i}$. For Condition (2) it suffices to construct a DFA recognizing the words $t_{1} \ldots t_{m} u$ where for all $i \leqslant m, t_{i}$ is a literal of $C_{i}$ and $u$ is an arbitrary word ( $u$ is necessary since some variables might not appear in the prefix, preventing the word from coding an assignment). The problem with these two template DFAs is that they do not verify Condition (3). To solve this issue, we add loops on their states in order to generate as many copies of infixes as necessary to make two words coding the same assignment indistinguishable wrt. the class LTT.

We begin by giving $\mathcal{A}_{1}$, of which a graphical representation is shown in Figure 5. It recognizes $L_{1}$, with the marked initial and final states.
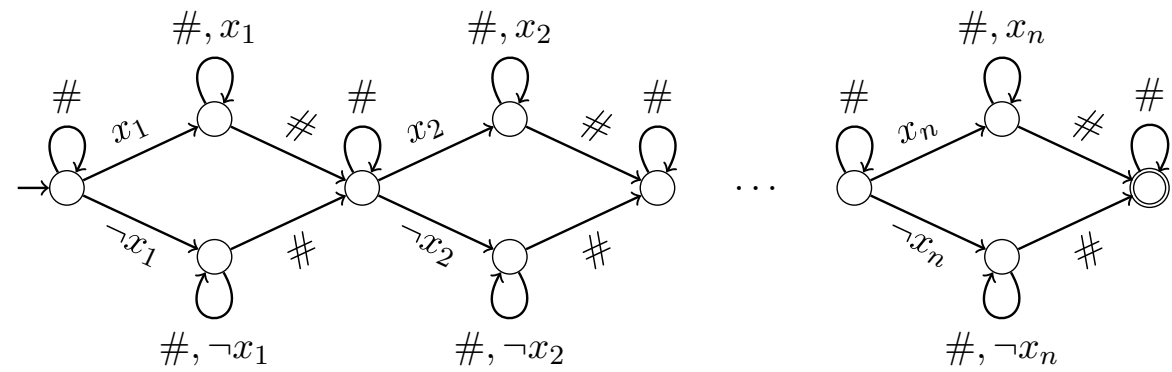

Figure 5: Representation of $\mathcal{A}_{1}$

By definition, $\mathcal{A}_{1}$ verifies Condition (11). We now define $\mathcal{A}_{2}$ as a sequence of $m$ subautomata, each one corresponding to a clause $C$ in $\mathcal{C}$. Intuitively, if $C=x_{i} \vee x_{j} \vee \neg x_{k}$, the subautomata selects a label within $\left\{x_{i}, x_{j}, \neg x_{k}\right\}$. This means that if this word encodes an assignment, it must satisfy all clauses in $\mathcal{C}$. We give a graphical representation of $\mathcal{A}_{2}$ in Figure 6 (it recognizes $L_{2}$, with the marked initial and final states).

By definition, $\mathcal{A}_{2}$ verifies Condition (2). It remains to verify that Condition (3) holds.

Lemma 6.9. If there are words $w_{1}, w_{2}$ accepted by $\mathcal{A}_{1}, \mathcal{A}_{2}$ that encode the same assignment, then for all $k, d \in \mathbb{N}$, they can be chosen such that $w_{1} \equiv_{k}^{d} w_{2}$.

Proof. This is done by using the loops in $\mathcal{A}_{1}, \mathcal{A}_{2}$ to generate as many copies of the $k$-profiles in $w_{1}, w_{2}$ as needed in order to get words that are $\equiv_{k}^{d}$-equivalent.

We finish by proving that $\mathcal{C}$ is satisfiable if and only if $L_{1}, L_{2}$ are not LTT-separable. Assume first that $L_{1}$ and $L_{2}$ are not LTT-separable. By Fact 6.8, this means that there exist $w_{1} \in L_{1}$ and $w_{2} \in L_{2}$ sharing the same alphabet. By Condition (11), $w_{1}$ encodes an assignment. Therefore, $w_{2}$ (which has the same alphabet) encodes the same assignment which satisfies $\mathcal{C}$ by Condition (2). Hence $\mathcal{C}$ is satisfiable.

Conversely, assume that $\mathcal{C}$ is satisfiable. We prove that for all $k, d \in \mathbb{N}, L_{1}, L_{1}$ are not LTT $[k, d]$-separable. Set $k, d \in \mathbb{N}$ and consider an assignment of truth values satisfying $\mathcal{C}$. 

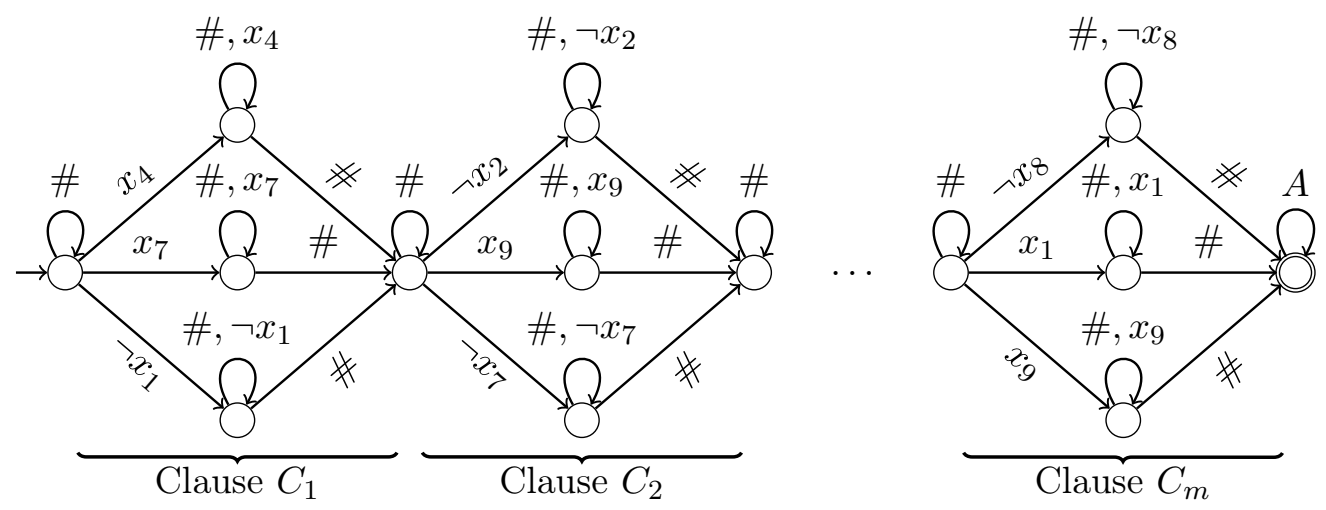

Figure 6: Representation of $\mathcal{A}_{2}$, for $C_{1}=x_{4} \vee x_{7} \vee \neg x_{1}, C_{2}=\neg x_{2} \vee x_{9} \vee \neg x_{7}, C_{m}=$ $\neg x_{8} \vee x_{1} \vee x_{9}$

By Conditions (11) and (2), there must exist $w_{1}, w_{2}$ accepted by $\mathcal{A}_{1}, \mathcal{A}_{2}$ that both encode this assignment. It follows from Lemma 6.9 that $w_{1}, w_{2}$ can be chosen such that $w_{1} \equiv_{k}^{d} w_{2}$. Therefore, $L_{1}, L_{2}$ are not LTT $[k, d]$-separable, which terminates the proof.

\section{The Case of Context-Free Languages}

In order to prove decidability of LTT-separability for regular languages, we needed three ingredients: Parikh's Theorem, decidability of Presburger Arithmetic and Item (2) in Theorem 5.1. Since Parikh's Theorem holds not only for regular languages but also for contextfree languages, we retain at least two of the ingredients in the context-free setting.

In particular, we can reuse the argument of Section 5 to prove that once the size $k$ of the infixes is fixed, separability by LTT is decidable for context-free languages. For any fixed $k \in \mathbb{N}$, we write $\operatorname{LTT}[k]=\bigcup_{d \in \mathbb{N}} \operatorname{LTT}[k, d]$.

Theorem 7.1. Let $L_{1}, L_{2}$ be context-free languages and $k \in \mathbb{N}$. It is decidable whether $L_{1}, L_{2}$ are LTT $[k]$-separable.

An interesting consequence of Theorem 7.1 is that LTT[1]-separability of context-free languages is decidable. A language is LTT[1] if and only if it can be defined by a first-order logic formula that can only test equality between positions, but not ordering. This result is surprising since membership of a context-free language in this class is undecidable. We give a proof of this fact below, which is a simple adaptation of the proof of Greibach's Theorem (which is in particular used to prove that regularity of a context-free language is undecidable).

Theorem 7.2. Let $L$ be a context-free language. It is undecidable whether $L \in \mathrm{LTT}[1]$.

Proof. We reduce universality of context-free languages to this membership problem. Let $L$ be a context-free language over $A$ and let \# $\notin A$. Let $K \notin$ LTT[1] be some context-free language and set $L_{1}=\left(K \cdot \# \cdot A^{*}\right) \cup\left(A^{*} \cdot \# \cdot L\right)$. Clearly, a context-free grammar for $L_{1}$ can be computed from a context-free grammar for $L$. We show that $L=A^{*}$ if and only if $L_{1} \in \mathrm{LTT}[1]$. 
If $L=A^{*}$, then $L_{1}=A^{*} \cdot \# \cdot A^{*} \in \operatorname{LTT}[1]$. Conversely, assume that $L_{1} \in \operatorname{LTT}[1]$, and suppose by contradiction that $L \neq A^{*}$. Pick $w \in A^{*}$ such that $w \notin L$. By definition, $K=\left\{u \mid u \# w \in L_{1}\right\}$. One can verify that LTT[1] is closed under right residuals. Therefore, $K=L_{1}(\# w)^{-1} \in \operatorname{LTT}[1]$ which is a contradiction by definition of $K$.

These two results may seem contradictory. Indeed in the setting of regular languages, membership can be reduced to separability (a language belongs to a class if the class can separate it from its complement). However, context-free languages are not closed under complement, which makes the reduction false in this larger setting.

An interesting question is whether decidability extends to full LT- and LTT-separability of context-free languages. This would also be surprising since membership of a context-free language in LT or LTT are undecidable problems. Such a result would require to generalize our third ingredient, Item (22) in Theorem 5.1, to context-free languages. This means that we would need a method for computing a bound on the size of the infixes that a potential separator has to consider. It turns out that this is not possible.

Theorem 7.3. Let $L_{1}, L_{2}$ be context-free languages. It is undecidable whether $L_{1}, L_{2}$ are LT-separable. It is undecidable whether $L_{1}, L_{2}$ are LTT-separable.

It was already known [41 that separability by a regular language is undecidable for context-free languages. The proof of Theorem 7.3 is essentially the same since the reduction provided in [41] actually works for any class of regular separators that contains all languages of the form $K_{1} A^{*} \cup K_{2}$ where $K_{1}, K_{2}$ are finite languages. Since this is clearly the case for both LT and LTT, Theorem 7.3 follows. For the sake of completeness, we provide a version of this proof tailored to LT and LTT below.

Proof of Theorem 7.3. The proof is done by reduction of the halting problem on Turing machines to LT-separability and LTT-separability. The reduction is the same for both LT and LTT and is essentially a rewriting of a proof of [41.

Consider a deterministic Turing machine $\mathcal{M}$. We prove that it is possible to compute context-free languages $L_{1}, L_{2}$ from $\mathcal{M}$ such that $\mathcal{M}$ halts on the empty input if and only if $L_{1}, L_{2}$ are LT-separable, if and only if $L_{1}, L_{2}$ are LTT-separable.

Let $A$ be the alphabet of $\mathcal{M}$, let $Q$ be its set of states, and let $B=A \cup(A \times Q) \cup\{\#, \gamma\}$ where \#, $\gamma \notin A$. As usual, we encode configurations of $\mathcal{M}$ as words in $A^{*} \cdot(A \times Q) \cdot A^{*} \subseteq B^{*}$ : the word $(u,(q, a), v)$ means that $\mathcal{M}$ is in state $q$, the tape holds $u \cdot a \cdot v$, and the head currently scans the distinguished $a$ position. Finally, if $w \in B^{*}$, we denote by $w^{R}$ the mirror image of $w$. We can now define the context-free languages $L_{1}, L_{2}$ over $B$. The language $L_{1}$ contains all words of the form:

$$
c_{1} \# c_{2}^{R} \# c_{3} \# c_{4}^{R} \cdots \# c_{2 k-1} \# c_{2 k}^{R} \# \gamma^{k}
$$

such that $c_{1}, \ldots, c_{2 k}$ are encodings of configurations of $\mathcal{M}$, and for all $i \leqslant k, c_{2 i-1} \vdash_{\mathcal{M}} c_{2 i}$ (i.e., $c_{2 i}$ is the configuration of $\mathcal{M}$ reached after one computation step from configuration $\left.c_{2 i-1}\right)$. Similarly, $L_{2}$ contains all words of the form:

$$
c_{1} \# c_{2}^{R} \# c_{3} \# c_{4}^{R} \cdots \# c_{2 k-1} \# c_{2 k}^{R} \# \gamma^{2 k}
$$

such that $c_{1}, \ldots, c_{2 k}$ are encodings of configurations of $\mathcal{M}, c_{1}$ is the initial configuration of $\mathcal{M}$ starting with an empty input and for all $i \leqslant k-1, c_{2 i} \vdash_{\mathcal{M}} c_{2 i+1}$. It is classical that $L_{1}, L_{2}$ are indeed context-free and that grammars for $L_{1}, L_{2}$ can be computed from $\mathcal{M}$. We 
now make a simple observation about prefixes that are common to both languages. Let $c_{1}, c_{2}, c_{3}, c_{4}, \ldots, c_{i-1}, c_{i}$ be a sequence of configurations and let $w \in B^{*}$ be the word

$$
\begin{array}{ll}
w=c_{1} \# c_{2}^{R} \# c_{3} \# c_{4}^{R} \cdots \# c_{2 k-1} \# c_{2 k}^{R} \# \quad & \text { (if } i=2 k), \\
w=c_{1} \# c_{2}^{R} \# c_{3} \# c_{4}^{R} \cdots \# c_{2 k-1} \# c_{2 k}^{R} \# c_{2 k+1} \# & (\text { if } i=2 k+1) .
\end{array}
$$

By definition of $L_{1}, L_{2}$, we have the following fact:

Fact 7.4. If $w$ is both a prefix of some word in $L_{1}$ and some word in $L_{2}$, then $c_{1}, c_{2}, \ldots, c_{i}$ are the first $i$ configurations of the run of $\mathcal{M}$ starting from the empty input. Moreover, if $i=2 k$ and

$$
c_{1} \# c_{2}^{R} \# c_{3} \# c_{4}^{R} \cdots \# c_{2 k-1} \# c_{2 k}^{R} \# c \#
$$

is a prefix of a word in $L_{2}$, then $c$ is configuration $(i+1)$ in the run. Symmetrically, if $i=2 k+1$ and

$$
c_{1} \# c_{2}^{R} \# c_{3} \# c_{4}^{R} \cdots \# c_{2 k-1} \# c_{2 k}^{R} \# c_{2 k+1} \# c^{R} \#
$$

is a prefix of a word in $L_{1}$, then $c$ is configuration $(i+1)$ in the run.

It remains to prove that this is indeed a reduction, i.e., that $\mathcal{M}$ halts on the empty input if and only if $L_{1}, L_{2}$ are LT-separable, if and only if $L_{1}, L_{2}$ are LTT-separable. Assume first that $\mathcal{M}$ does not halt on empty input. This means that the run of $\mathcal{M}$ is an infinite sequence of configurations $c_{1}, c_{2}, c_{3}, \ldots$ By definition of $L_{1}, L_{2}$, for all $k \in \mathbb{N}$ :

$$
\begin{array}{ll}
c_{1} \# c_{2}^{R} \# c_{3} \# c_{4}^{R} \cdots \# c_{2 k-1} \# c_{2 k}^{R} \# \gamma^{k} & \in L_{1}, \\
c_{1} \# c_{2}^{R} \# c_{3} \# c_{4}^{R} \cdots \# c_{2 k-1} \# c_{2 k}^{R} \# \gamma^{2 k} & \in L_{2} .
\end{array}
$$

It is then easy to deduce that $L_{1}, L_{2}$ cannot be separated by a LT or LTT language (actually not even by a regular language).

Conversely, assume that $\mathcal{M}$ halts on the empty input within $\ell$ steps, i.e., $c_{1} \vdash_{\mathcal{M}} c_{2} \vdash_{\mathcal{M}}$ $\ldots \vdash_{\mathcal{M}} c_{\ell}$ and $c_{\ell}$ is the halting configuration. Before defining an LT separator, we observe that sufficiently long prefixes of sufficiently long words of $L_{1}, L_{2}$ are distinct.

Lemma 7.5. Let $w_{1} \in L_{1}$ and $w_{2} \in L_{2}$ of length greater than $(\ell+1)^{2}+2(\ell+1)$ and let $u_{1}, u_{2}$ be the prefixes of length $\ell(\ell+1)+2 \ell+1$ of $w_{1}, w_{2}$, respectively. Then $u_{1} \neq u_{2}$.

Proof. We proceed by contradiction. Assume $u_{1}=u_{2}$ and let $u$ be the largest prefix of $u_{1}=u_{2}$ of the form

$$
\begin{aligned}
& u=c_{1} \# c_{2}^{R} \# c_{3} \# c_{4}^{R} \cdots \# c_{i-1} \# c_{i}^{R} \#, \quad \text { or } \\
& u=c_{1} \# c_{2}^{R} \# c_{3} \# c_{4}^{R} \cdots \# c_{i-1}^{R} \# c_{i} \# \quad \text { depending on whether } i \text { is even or not. }
\end{aligned}
$$

By definition $u_{1}=u_{2}=u \cdot v$, where $v$ can be of the form $\gamma^{j}$ with $j \leqslant i$ or is the prefix of $c$ or $c^{R}$ for some configuration $c$ of $\mathcal{M}$.

Assume first that $v=\gamma^{j}$ with $j \leqslant i$. By Fact $7.4, c_{1}, c_{2}, c_{3}, c_{4}, \ldots, c_{i-1}, c_{i}$ are the first $i$ configurations of the run of $\mathcal{M}$ starting from the empty input. Since $\mathcal{M}$ halts in $\ell$ steps, this means that $i \leqslant \ell$ and that each configuration $c_{i}$ is of length at most $\ell+1$. It follows that $u$ is of length at most $\ell(\ell+1)+\ell$. Therefore, $u_{1}=u_{2}$ is of length at most $\ell(\ell+1)+\ell+j \leqslant \ell(\ell+1)+\ell+i \leqslant \ell(\ell+1)+2 \ell$, in contradiction with the definition of $u_{1}, u_{2}$.

Assume finally that $v$ is the prefix of $c$ or $c^{R}$ for some configuration $c$ of $\mathcal{M}$. By Fact 7.4, $c$ is so that $c_{1}, c_{2}, c_{3}, c_{4}, \ldots, c_{i-1}, c_{i}, c$ are the first $i+1$ configurations of the run of $\mathcal{M}$ starting from the empty input. Since $\mathcal{M}$ halts in $\ell$ steps, this means that $i+1 \leqslant \ell$ and that each 
configuration is of length at most $\leqslant \ell+1$. It follows that $u_{1}=u_{2}$ is of length at most $\ell(\ell+1)+\ell$ which is again a contradiction.

Let $K_{1}$ be the language of words of $L_{1}$ of length less than $\ell(\ell+1)+2 \ell+1$. Similarly, let $K_{2}$ be the set of prefixes of length $\ell(\ell+1)+2 \ell+1$ of words in $L_{1}$. Finally, set $L=K_{1} \cup K_{2} \cdot B^{*}$. By definition, $K_{1}, K_{2}$ are finite languages, hence $L$ is clearly LT (and therefore LTT). It remains to prove that $L$ is a separator.

By definition, we have $L_{1} \subseteq L$. Let us prove that $L \cap L_{2}=\varnothing$. We proceed by contradiction: assume that there exists $w \in L \cap L_{2}$. If $w \in K_{1}$, then the contradiction is immediate because, by definition of $L_{1}, w$ must be in of the form

$$
c_{1} \# c_{2}^{R} \# c_{3} \# c_{4}^{R} \cdots \# c_{2 k-1} \# c_{2 k}^{R} \# \gamma^{k}
$$

Now, belonging to $L_{2}$ would require twice as many letters $\gamma$ at the end of the word. On the other hand, if $w \in K_{2} \cdot B^{*}$, then, by definition of $K_{2}$ there exists some word of length $\ell(\ell+1)+2 \ell+1$ that is both a prefix of a word in $L_{1}$ and a prefix of a word in $L_{2}$. This contradicts Lemma 7.5 .

We deduce that $L_{1}, L_{2}$ are LT-separable, and therefore also LTT-separable, which concludes the proof of Theorem 7.3 .

\section{Conclusion}

We have shown separation theorems for both LT and LTT. In both cases, we provide a decision procedure to test separability, running in CO-NEXPTIME and 2-EXPSPACE respectively. Another contribution is a description of possible separators, given by bounds defining them.

Several questions remain open in this line of research. A first one is to obtain tight complexity bounds for both classes. While we have CO-NEXPTime and 2-EXPSPACE upper bounds for LT and LTT respectively, we have only CO-NP lower bounds. The upper bounds rely on a reduction to the case $k=1$, i.e., a translation to the special case when the size of infixes is fixed to 1 . This translation is exponential wrt. the size of the input automata. Improving the upper bounds would likely require improving this reduction.

Another question is to generalize our techniques to other settings, and to obtain transfer results. First, one can consider other fragments for separability. For instance, if separation is decidable for some fragment of first-order logic, is it still decidable when adding some predicate to the logic, such as the successor? In the present paper, this is what we do for the specific case of LTT, whose corresponding logic, FO $(+1)$, is obtained by adding the successor to first-order logic with only equality on positions and alphabetic predicates. Another natural example is adding modulo predicates, which would allow to treat LTT + MOD for instance, the generalization of LTT in which infixes can now also be counted modulo constants. Generalizing our results to more complex structures such as trees would also be interesting. However, in the setting of trees, while decidable characterizations are known for both LT and LTT [3, 31], no delay theorem is known. This makes separation a challenging problem as our techniques rely on a generalization of this theorem.

Acknowledgement. We thank the referees for their careful reading and helpful suggestions. 


\section{REFERENCES}

[1] J. Almeida. Some algorithmic problems for pseudovarieties. Publ. Math. Debrecen, 54(suppl.):531-552, 1999. Automata and formal languages, VIII (Salgótarján, 1996).

[2] D. Beauquier and J. E. Pin. Languages and scanners. Theoret. Comput. Sci., 84(1):3-21, 1991.

[3] M. Benedikt and L. Segoufin. Regular Languages Definable in FO and FOmod. ACM Transactions of Computational Logic, 11(1), 2009.

[4] M. Bojańczyk. A new algorithm for testing if a regular language is locally threshold testable. Inf. Process. Lett., 104(3):91-94, 2007.

[5] I. Borosh and L. B. Treybig. Bounds on positive integral solutions of linear Diophantine equations. Proc. Amer. Math. Soc., 55(2):299-304, 1976.

[6] J. Brzozowski and I. Simon. Characterizations of locally testable events. Discrete Mathematics, 4(3):243271, 1973.

[7] J. R. Büchi. Weak second-order arithmetic and finite automata. Mathematical Logic Quarterly, 6(16):66-92, 1960.

[8] Y.-F. Chen, A. Farzan, E. M. Clarke, Y.-K. Tsay, and B.-Y. Wang. Learning minimal separating DFA's for compositional verification. In TACAS'09, volume 5505 of Lect. Notes Comp. Sci., pages 31-45. Springer, 2009.

[9] C. Choffrut, F. D'Alessandro, and S. Varricchio. On the separability of sparse context-free languages and of bounded rational relations. Theoret. Comput. Sci., 381(1-3):274-279, 2007.

[10] C. Choffrut and S. Grigorieff. Separability of rational relations in $A^{*} \times \mathbb{N}^{m}$ by recognizable relations is decidable. Inf. Process. Lett., 99(1):27-32, 2006.

[11] J. C. Costa. Free profinite locally idempotent and locally commutative semigroups. J. Pure Appl. Algebra, 163(1):19-47, 2001.

[12] J. C. Costa and C. Nogueira. Complete reducibility of the pseudovariety LSl. Internat. J. Algebra Comput., 19(02):247-282, 2009.

[13] W. Czerwinski, W. Martens, and T. Masopust. Efficient separability of regular languages by subsequences and suffixes. In Proc. of ICALP'13, volume 7966 of Lect. Notes Comp. Sci., pages 150-161. Springer, 2013.

[14] C. C. Elgot. Decision problems of finite automata design and related arithmetics. Trans. Amer. Math. Soc., 98(1):21-51, 1961.

[15] S. Ginsburg and E. H. Spanier. Semigroups, Presburger formulas and languages. Pacific Journal of Mathematics, 16(2):285-296, 1966.

[16] A. Gupta, K. McMillan, and Z. Fu. Automated assumption generation for compositional verification. Formal Methods in System Design, 32(3):285-301, 2008.

[17] K. Henckell. Pointlike sets: the finest aperiodic cover of a finite semigroup. J. Pure Appl. Algebra, 55(1-2):85-126, 1988.

[18] T. A. Henzinger, R. Jhala, R. Majumdar, and K. L. McMillan. Abstractions from proofs. In Proc. of POPL'04, pages 232-244. ACM, 2004.

[19] H. B. Hunt, III. Decidability of grammar problems. J. ACM, 29(2):429-447, 1982.

[20] S. Kim, R. McNaughton, and R. McCloskey. A polynomial time algorithm for the local testability problem of deterministic finite automata. In Algorithms and Data Structures, number 382 in Lect. Notes Comp. Sci., pages 420-436. Springer, 1989.

[21] J. Leroux. Vector addition systems reachability problem (a simpler solution). In The Alan Turing Centenary Conference, Turing-100, volume 10, pages 214-228, 2012.

[22] M. Leucker. Learning meets verification. In Proc. of FMCO'06, pages 127-151. Springer, 2007.

[23] E. W. Mayr. An algorithm for the general Petri net reachability problem. SIAM J. Comput., 13(3):441460, 1984.

[24] K. L. McMillan. Applications of Craig interpolants in model checking. In Proc. of TACAS'05, pages 1-12. Springer, 2005.

[25] R. McNaughton. Algebraic decision procedures for local testability. Math. Systems Theory, 8(1):60-76, 1974.

[26] R. McNaughton and S. Papert. Counter-free automata. The M.I.T. Press, 1971.

[27] R. J. Parikh. On context-free languages. J. ACM, 13(4):570-581, 1966.

[28] J.-E. Pin. The expressive power of existential first order sentences of Büchi's sequential calculus. In Proc. of ICALP'96, number 1099 in Lect. Notes Comp. Sci., pages 300-311. Springer, 1996. 
[29] J. E. Pin. Syntactic semigroups. In Handbook of language theory, Vol. I, pages 679-746. Springer, 1997.

[30] J. E. Pin. Expressive power of existential first-order sentences of Büchi's sequential calculus. Discrete Math., 291(1-3):155-174, 2005.

[31] T. Place and L. Segoufin. A decidable characterization of locally testable tree languages. Logical Methods in Computer Science, 7(4), 2011.

[32] T. Place, L. van Rooijen, and M. Zeitoun. Separating regular languages by piecewise testable and unambiguous languages. In Proc. of MFCS'13, number 8087 in Lect. Notes Comp. Sci., pages 729-740. Springer, 2013.

[33] T. Place and M. Zeitoun. Going higher in the first-order quantifier alternation hierarchy on words. In ICALP'14, 2014.

[34] T. Place and M. Zeitoun. Separating regular languages with first-order logic. In CSL-LICS'14, 2014.

[35] C. R. Reddy and D. W. Loveland. Presburger arithmetic with bounded quantifier alternation. In STOC'78, pages 320-325. ACM, 1978.

[36] M. P. Schützenberger. On finite monoids having only trivial subgroups. Information and Control, 8(2):190-194, 1965.

[37] H. Seidl, T. Schwentick, A. Muscholl, and P. Habermehl. Counting in trees for free. In ICALP'O4, volume 3142 of Lect. Notes Comp. Sci., pages 1136-1149. Springer, 2004.

[38] B. Steinberg. On pointlike sets and joins of pseudovarieties. Internat. J. Algebra Comput., 8(2):203-231, 1998.

[39] B. Steinberg. A delay theorem for pointlikes. Sem. Forum, 63(3):281-304, 2001.

[40] H. Straubing. Finite semigroup varieties of the form $V * D$. J. Pure Appl. Algebra, 36(C):53-94, 1985.

[41] T. G. Szymanski and J. H. Williams. Noncanonical extensions of bottom-up parsing techniques. SIAM J. Comput., 5(2):231-250, 1976.

[42] D. Thérien and A. Weiss. Graph congruences and wreath products. J. Pure Appl. Algebra, 36:205-215, 1985.

[43] W. Thomas. Classifying regular events in symbolic logic. J. Comput. Syst. Sci., 25(3):360-376, 1982.

[44] A. N. Trahtman. An algorithm to verify local threshold testability of deterministic finite automata. In Automata Implementation, number 2214 in Lect. Notes Comp. Sci., pages 164-173. Springer, 2001.

[45] B. A. Trakhtenbrot. Finite automata and logic of monadic predicates. Doklady Akademii Nauk SSSR, 149:326-329, 1961. In Russian.

[46] M. Y. Vardi and P. Wolper. An automata-theoretic approach to automatic program verification. In Proc. of LICS'86, pages 332-344. IEEE Computer Society, 1986.

[47] K. N. Verma, H. Seidl, and T. Schwentick. On the complexity of equational Horn clauses. In Proc. of CADE'05, volume 3632 of Lect. Notes Comp. Sci., pages 337-352, 2005.

[48] J. von zur Gathen and M. Sieveking. A bound on solutions of linear integer equalities and inequalities. Proc. Amer. Math. Soc., 72:155-158, 1978.

[49] Y. Zalcstein. Locally testable languages. J. Comput. Syst. Sci., 6(2):151-167, 1972.

This work is licensed under the Creative Commons Attribution-NoDerivs License. To view a copy of this license, visit http://creativecommons.org/licenses/by-nd/2.0/ or send a letter to Creative Commons, 171 Second St, Suite 300, San Francisco, CA 94105, USA, or Eisenacher Strasse 2, 10777 Berlin, Germany 